\title{
Dynamic interactions of an integrated vehicle-electromagnetic energy harvester-tire system subject to uneven road excitations
}

\author{
Jing Tang Xing ${ }^{1} \cdot$ Zhe Sun $^{1} \cdot$ Sulian Zhou ${ }^{1,2} \cdot$ Mingyi Tan $^{1}$
}

Received: 17 October 2016 / Accepted: 28 December 2016 / Published online: 10 March 2017

(C) The Author(s) 2017. This article is published with open access at Springerlink.com

\begin{abstract}
An investigation is undertaken of an integrated mechanical-electromagnetic coupling system consisting of a rigid vehicle with heave, roll, and pitch motions, four electromagnetic energy harvesters and four tires subject to uneven road excitations in order to improve the passengers' riding comfort and harvest the lost engine energy due to uneven roads. Following the derived mathematical formulations and the proposed solution approaches, the numerical simulations of this interaction system subject to a continuous sinusoidal road excitation and a single ramp impact are completed. The simulation results are presented as the dynamic response curves in the forms of the frequency spectrum and the time history, which reveals the complex interaction characteristics of the system for vibration reductions and energy harvesting performance. It has addressed the coupling effects on the dynamic characteristics of the integrated system caused by: (1) the natural modes and frequencies of the vehicle; (2) the vehicle rolling and pitching motions; (3) different road excitations on four wheels; (4) the time delay of a road ramp to impact both the front and rear wheels, etc., which cannot be tackled by an often used quarter vehicle model. The guidelines for engineering applications are given. The developed coupling model and the revealed concept provide a means with analysis idea to investigate the details of four energy harvester motions for electromagnetic suspension designs in order to replace the current passive vehicle isolators and to harvest the lost engine energy. Potential further research directions are suggested for readers to consider in the future.
\end{abstract}

Jing Tang Xing

jtxing@soton.ac.uk

1 Fluid Structure Interactions Group, FEE, University of Southampton, Southampton SO17 1BJ, UK

2 College of Shipbuilding Engineering, Harbin Engineering University, Harbin 150001, China
Keywords Vibration-energy-harvesters · Electromagnetic suspensions - Mechanical electromagnetic interactions . Vehicle dynamics · Vibration isolations

\section{Introduction}

Environmental concerns and fuel price increases have accelerated the global trend toward the following two important research directions. One is to seek new green energy resources, such as wind/wave energy harvested by different energy converters [1-16], where the fundamental principle includes two main points: (1) to design an energy harvesting device excited by winds or waves to undergo as large mechanical motions as possible; (2) to convert the mechanical energy into a type of storable energy, such as electric or chemical ones, etc. To obtain the large motions of an energy harvest device, the mechanisms of resonances in linear dynamic systems and the flutter of aerofoils, which are harmful vibrations to be avoided in traditional dynamic designs, are in a reverse case to be used to obtain the expended large motions of the energy harvesting device [12-16]. The review papers [4,16] provide more details on various wave/wind energy harvesters as well as many available references. The interested readers may refer to them for more information on this topic. Another research direction is to develop low-emission and fuel-efficient designs to reduce energy consumption. In this direction, the majority of current publications aim to harvest the energy of vibrations in various transportation modes: airplanes, trains, and vehicles, etc. These vibrations are caused by different operation environments, for example, the vehicle vibrations excited by uneven roads, airplane cabin noises caused by engines. The energy of this type of vibrations is the output of burning fuels in their engines, but normally is dissipated by the damping of the transport and the environment, 
which is an energy loss. As indicated in Ref. [17], during the everyday usage of an automobile, only $10 \%-16 \%$ of the fuel energy is used to drive the vehicle to overcome the resistance from the road friction and the air drag, so that about $85 \%$ of the fuel energy is dissipated by the shock absorbers of the vehicle suspension system. Therefore, there have been many research papers theoretically, numerically and experimentally dealing with different designs to harvest this type of vibration energy.

In vehicle designs, applications of passive viscous shock absorbers in parallel with suspension springs aim to reduce vibrations and to achieve better riding/road handling qualities. Active suspensions have been explored [18], but their operation requires a significant amount of energy, which limits their wide implementations. To overcome this, initial theoretical researches on the feasibility to harvest the vibration energy from vehicle suspensions were reported $[19,20]$, which examined the possibility of using a permanent magnetic motor as mechanical dampers for vehicles to dissipate their vibration energy. Following this initial idea, many publications on this topic have been reported. References such as [21-36] presented some concepts, investigations, numerical simulations, various types of absorber designs, experimental tests, piezoelectric transducers [28], as well as the involved nonlinear behaviour [31]. It has been proposed to adopt regenerative suspensions in harvesting the vehicle lost engine energy while reducing its vibrations for better ride performance. The more details on those researches one can be referred to the comprehensive review papers $[29,34]$ as well as a series of research reports [32-36]. The company, Perpetuum [37], specialises in vibrational energy harvesting devices and has produced a range of energy harvesting nodes on rail applications and several thousand units are currently mounted on UK and European trains.

In the available publications to analyse the performance of energy harvesting absorbers, the fundamental concept model is a quarter vehicle one, which has omitted the interactions between vehicle vibration modes, four absorbers as well as different uneven road excitations to four wheels. It has been noticed that a full vehicle schematic model with seven degrees of freedom (DOF) is proposed in Ref. [30], but not for energy harvesting investigations, so that there is no interaction results on vehicle vibration reduction and energy harvesting performance given from this model. This paper develops an integrated mechanical-electrical interaction system consisting of a rigid vehicle with three main important degrees of freedom to describe its heave, roll and pitch motions, four electro-magnetic regenerative suspensions, and four tires, each of them modelled by its stiffness and damper, subjected to uneven road excitations to study its complex coupling dynamic mechanisms. This model is able to reveal the effects of uneven road, the damping of the energy harvester, vehicle natural frequencies/modes, and driving speeds, etc. on the performance of the energy harvester to collect the vehicle vibration energy and at the same time to improve its ride quality.

\section{Mathematical model}

As shown in Fig. 1a, we consider the vehicle as a rigid frame, with the sprung mass $\bar{M}$ located at its mass center $O$ at which a vehicle body coordinate system $O-X Y Z$ with three unit coordinate vectors $\boldsymbol{i}_{X}, \boldsymbol{i}_{Y}$, and $\boldsymbol{i}_{Z}$ are fixed. This frame is supported by the four suspension units, of which, as shown by Fig. $1 \mathrm{~b}$, a representative unit $I(I=1,2,3,4)$ at point $\left(\hat{X}_{I}, \hat{Y}_{I}, \hat{Z}_{I}\right)$ consists of a suspension spring of stiffness $K_{I}$, an electromagnetic energy converter of damping $C_{I}$, wheelmagnetic body mass $m_{I}$, and wheel stiffness $k_{I}$ and damping $c_{I}$. The vehicle moves on an uneven road surface denoted by a height function $h\left(x_{I}, y_{I}\right)$ measured in a fixed absolute coordinate system $o-x y z$, of which the origin $o$ is located at point $O$ and its three axes are respectively parallel the three axes of the body coordinate system $O-X Y Z$ when the vehicle is in a stable state moving along an even road in the $o-x$ direction in a constant velocity $V$. Uneven road excitations to the four wheels will cause the vehicle vibrations, assuming only in the vertical direction, described by a vertical translation $Z$ of the mass center $O$, a roll rotation angle $\theta_{x}$ about axis $o-x$ and a pitch rotation angle $\theta_{y}$ about axis $o-y$, but neglecting the motions in the horizontal translations and yaw rotations about axis $o-z$. The roll and pitch inertias of the vehicle about the body system are denoted by $J_{x}$ and $J_{y}$, respectively. The motion $z_{I}$ of mass $m_{I}$ is also considered in the vertical direction with any horizontal motions neglected. The steady position of the system in the even road is chosen as our reference positon to measure the small vibrations of the vehicle, based on which we can derive the dynamic equations of the system as follows.

\subsection{Geometrical parameters and dynamical variables}

We adopt the following vectors and matrices of the geometrical parameters and dynamical variables to derive the governing equations describing the interaction dynamics of the integrated system.

\section{Rotation vector of vehicle body}

$\boldsymbol{\theta}=\theta_{X} \boldsymbol{i}_{X}+\theta_{Y} \boldsymbol{i}_{Y}, \quad \boldsymbol{\theta}=\left[\begin{array}{ll}\theta_{X} & \theta_{Y}\end{array}\right]^{\mathrm{T}}$. 

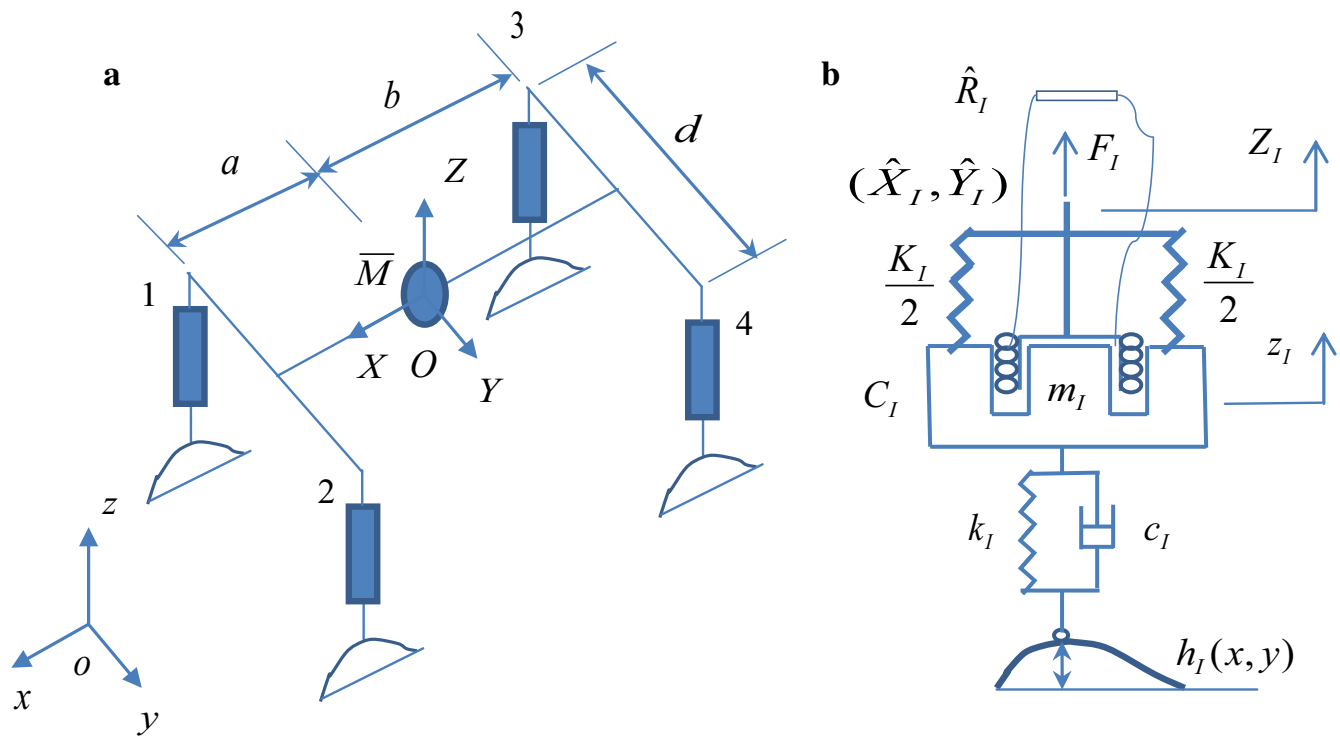

Fig. 1 a Scheme of integrated vehicle-energy harvester-tire interaction system with four electro-magnetic suspension units fixed at points $I=$ $1,2,3,4$. b The details of a representative suspension unit $I$

Position vector of suspension unit I

$\hat{\boldsymbol{R}}_{I}=\hat{X}_{I} \boldsymbol{i}_{X}+\hat{Y}_{I} \boldsymbol{i}_{Y}, \quad \hat{\boldsymbol{R}}_{I}=\left[\hat{X}_{I} \hat{Y}_{I}\right]^{\mathrm{T}}$.

\section{Displacement $Z_{I}$ and velocity $\dot{Z}_{I}$ of suspension unit I}

The vehicle body undergoes a small rigid motion consisting of the vertical translation $Z$ and the small rotation denoted by Eq. (1), so that the displacement $Z_{I}$ of suspension unit $I$ is calculated as

$$
\begin{aligned}
& Z_{I}=Z+\left\{\boldsymbol{\theta} \times \hat{\boldsymbol{R}}_{I}\right\}_{Z}=Z+\theta_{X} \hat{Y}_{I}-\theta_{Y} \hat{X}_{I} \\
& =Z+\left[\hat{Y}_{I}-\hat{X}_{I}\right]\left[\begin{array}{l}
\theta_{X} \\
\theta_{Y}
\end{array}\right], \\
& \boldsymbol{Z}_{s}=\boldsymbol{I}_{s} Z+\boldsymbol{X}_{s}^{\mathrm{T}} \boldsymbol{\theta}, \\
& \boldsymbol{Z}_{s}=\left[\begin{array}{llll}
Z_{1} & Z_{2} & Z_{3} & Z_{4}
\end{array}\right]^{\mathrm{T}}, \quad \boldsymbol{I}_{s}=\left[\begin{array}{llll}
1 & 1 & 1 & 1
\end{array}\right]^{\mathrm{T}}, \\
& \boldsymbol{X}_{S}=\left[\begin{array}{llll}
\hat{Y}_{1} & \hat{Y}_{2} & \hat{Y}_{3} & \hat{Y}_{4} \\
-\hat{X}_{1}-\hat{X}_{2}-\hat{X}_{3} & -\hat{X}_{4}
\end{array}\right] \text {, }
\end{aligned}
$$

from which, it follows its time derivatives

$$
\begin{aligned}
& \dot{Z}_{I}=\dot{Z}+\left[\hat{Y}_{I}-\hat{X}_{I}\right]\left[\begin{array}{c}
\dot{\theta}_{X} \\
\dot{\theta}_{Y}
\end{array}\right], \\
& \ddot{Z}_{I}=\ddot{Z}+\left[\hat{Y}_{I}-\hat{X}_{I}\right]\left[\begin{array}{c}
\ddot{\theta}_{X} \\
\ddot{\theta}_{Y}
\end{array}\right], \\
& \dot{\boldsymbol{Z}}_{s}=\boldsymbol{I}_{s} \dot{Z}+\boldsymbol{X}_{s}^{\mathrm{T}} \dot{\boldsymbol{\theta}}, \quad \ddot{\boldsymbol{Z}}_{s}=\boldsymbol{I}_{s} \ddot{Z}+\boldsymbol{X}_{s}^{\mathrm{T}} \ddot{\boldsymbol{\theta}} .
\end{aligned}
$$

Suspension force $F_{I}$ of unit I

$\boldsymbol{F}_{I}=F_{I} i_{Z}, F_{I}=K_{I}\left(Z_{I}-z_{I}\right)+C_{I}\left(\dot{Z}_{I}-\dot{z}_{I}\right)$,

$$
\begin{aligned}
& \boldsymbol{F}_{s}=\left[\begin{array}{llll}
F_{1} & F_{2} & F_{3} & F_{4}
\end{array}\right]^{\mathrm{T}}, \\
& \boldsymbol{F}_{s}=\boldsymbol{K}_{s}\left(\boldsymbol{Z}_{s}-\boldsymbol{z}\right)+\boldsymbol{C}_{s}\left(\dot{\boldsymbol{Z}}_{s}-\dot{\boldsymbol{z}}\right) \\
& =\boldsymbol{K}_{s}\left(\boldsymbol{I}_{s} Z+\boldsymbol{X}_{s}^{\mathrm{T}} \boldsymbol{\theta}-\boldsymbol{z}\right)+\boldsymbol{C}_{s}\left(\boldsymbol{I}_{s} \dot{\boldsymbol{Z}}+\boldsymbol{X}_{s}^{\mathrm{T}} \dot{\boldsymbol{\theta}}-\dot{\boldsymbol{z}}\right), \\
& \boldsymbol{K}_{s}=\operatorname{diag}\left(K_{I}\right), \boldsymbol{C}_{s}=\operatorname{diag}\left(C_{I}\right), \boldsymbol{z}=\left[\begin{array}{llll}
z_{1} & z_{2} & z_{3} & z_{4}
\end{array}\right]^{\mathrm{T}} .
\end{aligned}
$$

\subsection{Dynamical equations of vehicle body}

Using Newton's second law, we obtain the following dynamic equations governing the motions of the system.

Vertical translation of vehicle body

$\bar{M} \ddot{Z}+\sum_{I=1}^{4} F_{I}=\bar{M} \ddot{Z}+\boldsymbol{I}_{s}^{\mathrm{T}} \boldsymbol{K}_{s}\left(\boldsymbol{Z}_{s}-\boldsymbol{z}\right)+\boldsymbol{I}_{s}^{\mathrm{T}} \boldsymbol{C}_{s}\left(\dot{\boldsymbol{Z}}_{s}-\dot{\boldsymbol{z}}\right)=0$.

Rotation of vehicle body

$\boldsymbol{J} \ddot{\boldsymbol{\theta}}+\sum_{I=1}^{4}\left(\hat{\boldsymbol{R}}_{I} \times \boldsymbol{F}_{I}\right)=\boldsymbol{J} \ddot{\boldsymbol{\theta}}+\boldsymbol{X}_{S} \boldsymbol{F}_{s}=\mathbf{0}, \quad \boldsymbol{J}=\left[\begin{array}{cc}J_{X} & 0 \\ 0 & J_{Y}\end{array}\right]$.

Vertical motion of wheel I

$$
\begin{aligned}
& \boldsymbol{m} \ddot{\boldsymbol{z}}-\boldsymbol{F}_{s}+\boldsymbol{k}_{S}(\boldsymbol{z}-\boldsymbol{h})+\boldsymbol{c}_{s}(\dot{\boldsymbol{z}}-\dot{\boldsymbol{h}})=\mathbf{0}, \\
& \boldsymbol{m}=\operatorname{diag}\left(m_{I}\right), \boldsymbol{k}_{s}=\operatorname{diag}\left(k_{I}\right), \\
& \boldsymbol{c}_{s}=\operatorname{diag}\left(c_{I}\right), \boldsymbol{h}=\left[\begin{array}{llll}
h_{1} & h_{2} & h_{3} & h_{4}
\end{array}\right]^{\mathrm{T}} .
\end{aligned}
$$

These equations can be represented by the following matrix form 


$$
\begin{aligned}
& M \ddot{Z}+C \dot{Z}+K Z=F, \\
& \boldsymbol{C}=\left[\begin{array}{ccc}
\boldsymbol{I}_{s}^{\mathrm{T}} \boldsymbol{C}_{s} \boldsymbol{I}_{s} & \boldsymbol{I}_{s}^{\mathrm{T}} \boldsymbol{C}_{s} \boldsymbol{X}_{s}^{\mathrm{T}} & -\boldsymbol{I}_{s}^{\mathrm{T}} \boldsymbol{C}_{s} \\
\boldsymbol{X}_{s} \boldsymbol{C}_{s} \boldsymbol{I}_{s} & \boldsymbol{X}_{s} \boldsymbol{C}_{s} \boldsymbol{X}_{s}^{\mathrm{T}} & -\boldsymbol{X}_{s} \boldsymbol{C}_{s} \\
-\boldsymbol{C}_{s} \boldsymbol{I}_{s} & -\boldsymbol{C}_{s} \boldsymbol{X}_{s}^{\mathrm{T}} & \boldsymbol{C}_{s}+\boldsymbol{c}_{s}
\end{array}\right], \\
& \boldsymbol{K}=\left[\begin{array}{ccc}
\boldsymbol{I}_{s}^{\mathrm{T}} \boldsymbol{K}_{s} \boldsymbol{I}_{s} & \boldsymbol{I}_{s}^{\mathrm{T}} \boldsymbol{K}_{s} \boldsymbol{X}_{s}^{\mathrm{T}} & -\boldsymbol{I}_{s}^{\mathrm{T}} \boldsymbol{K}_{s} \\
\boldsymbol{X}_{s} \boldsymbol{K}_{s} \boldsymbol{I}_{s} & \boldsymbol{X}_{s} \boldsymbol{K}_{s} \boldsymbol{X}_{s}^{\mathrm{T}} & -\boldsymbol{X}_{s} \boldsymbol{K}_{s} \\
-\boldsymbol{K}_{s} \boldsymbol{I}_{s} & -\boldsymbol{K}_{s} \boldsymbol{X}_{s}^{\mathrm{T}} & \boldsymbol{K}_{s}+\boldsymbol{k}_{s}
\end{array}\right], \\
& \boldsymbol{M}=\operatorname{diag}(\bar{M}, \quad \boldsymbol{J}, \quad \boldsymbol{m}), \quad \boldsymbol{Z}=\left[\begin{array}{c}
Z \\
\boldsymbol{\theta} \\
z
\end{array}\right], \\
& \boldsymbol{F}=\left[\begin{array}{l}
0 \\
\mathbf{0} \\
\boldsymbol{k}_{s} \boldsymbol{h}
\end{array}\right]+\left[\begin{array}{l}
0 \\
\mathbf{0} \\
\boldsymbol{c}_{s} \boldsymbol{h}
\end{array}\right]
\end{aligned}
$$

\subsection{Energy flow equation}

Following the energy flow theory [38] and pre-multiplying Eq. (9) by the velocity vector $\dot{\boldsymbol{Z}}^{\mathrm{T}}$, we can derive the energy flow equation of the system in the form

$$
\begin{aligned}
& \dot{T}+\dot{D}+\dot{\Pi}=P, \\
& T=\frac{1}{2} \dot{\boldsymbol{Z}}^{\mathrm{T}} \boldsymbol{M} \dot{\boldsymbol{Z}}, \quad D=\int_{0}^{t} \dot{\boldsymbol{Z}}^{\mathrm{T}} \boldsymbol{C} \dot{\boldsymbol{Z}} \mathrm{d} t, \quad \Pi=\frac{1}{2} \boldsymbol{Z}^{\mathrm{T}} \boldsymbol{K} \boldsymbol{Z}, \\
& P=\dot{\boldsymbol{Z}}^{\mathrm{T}} \boldsymbol{F}=\dot{\boldsymbol{z}}^{\mathrm{T}}\left(\boldsymbol{k}_{s} \boldsymbol{h}+\boldsymbol{c}_{s} \dot{\boldsymbol{h}}\right)=\sum_{I=1}^{4} P_{I}, \\
& P_{I}=\dot{z}_{I}\left(k_{I} h_{I}+c_{I} \dot{h}_{I}\right) .
\end{aligned}
$$

Here, $T, D$, and $\Pi$ represent the kinetic, dissipated/collected, and potential energy of the system, respectively, while $P$, the summation of each input power $P_{I}$ from wheel $I$, gives the total power input from the road excitation. Based on the energy conservation law, this input power, originally produced by the vehicle engine, vanishes if the vehicle is driving along an even road with $h_{I}=0$. Against the uneven road, the vehicle engine will output an extra power, which is a nonuseful power to cause vibrations and an uncomfortable riding environment for passengers. In current vehicle designs, a passive suspension system is used to damp these vibrations in order to achieve riding comfort, which physically dissipates this output power by the suspension dampers, so that this power is totally wasted. In this research, we use the four electro-magnetic energy harvesting converters of damping $C_{I}$ to replace the passive suspension dampers in order to collect this part of engine energy and to reduce the vibrations caused by uneven road excitations at the same time.

For the characteristic time period $\hat{T}$ of the road function, the time averaged energy flow equation is given by

$$
\langle\dot{T}\rangle+\langle\dot{D}\rangle+\langle\dot{\Pi}\rangle=\langle P\rangle,
$$

where, for an arbitrary time-variable $A(t)$, such as $A(t)=$ $P(t)$, its time averaged value is defined as

$\langle A(t)\rangle=\frac{1}{\hat{T}} \int_{0}^{\hat{T}} A \mathrm{~d} t$.

\subsection{Energy harvesting converter and its collected power}

As shown in Fig. 1b, the energy harvesting converter $I$ consists of a magnetic body of intensity $B_{I}$ and an electric coil, of effective conduct length $l_{I}$, electrical inductance $L_{I}$, internal resistance $r_{I}$, and capacitance $\hat{C}_{I}$, moving across the electromagnetic field, so that an induced voltage $e_{I}$ at the two ends of the power collection device of resistance $\hat{R}_{I}$ is produced. Therefore, an electric charge $\hat{Q}_{I}$ with the corresponding current $\hat{I}_{I}$ is generated in the moving coil, from which an electromagnetic force $\hat{F}_{I}$ between the moving coil and the magnetic body is further produced to resist their relative motion. Based on the Laplace electromagnetic theorem and electrical equilibrium principle $[12,15,16,39,40]$, the following equations are established.

$e_{I}=B_{I} l_{I}\left(\dot{Z}_{I}-\dot{z}_{I}\right), \hat{F}_{I}=\hat{I}_{I} B_{I} l_{I}$.

The electric equilibrium equation of the circuit of total resistance $R_{I}=\hat{R}_{I}+r_{I}$ is given by

$e_{I}=L_{I} \ddot{\hat{Q}}_{I}+R_{I} \dot{\hat{Q}}+\hat{Q}_{I} / \hat{C}_{I}=L_{I} \dot{\hat{\hat{I}}}_{I}+R_{I} \hat{I}_{I}+\hat{Q}_{I} / \hat{C}_{I}, \hat{I}_{I}=\dot{\hat{Q}}_{I}$.

From Eqs. (13) and (14), we obtain the following relationship between the current and the mechanical motions,

$L_{I} \ddot{\hat{I}}_{I}+R_{I} \dot{\hat{I}}_{I}+\hat{I}_{I} / \hat{C}_{I}=B_{I} l_{I}\left(\ddot{Z}_{I}-\ddot{z}_{I}\right)$.

Equation (15) is coupled with Eq. (9), so that the integrated system is an electric-mechanical interaction system. Considering practical cases for which the capacitance $\hat{C}_{I}$ of the moving coil is normally quite large, and the inductance $L_{I}$ is small, especially for the low frequency road excitations, we can reduce Eq. (15) to

$R_{I} \hat{I}_{I}=B_{I} l_{I}\left(\dot{Z}_{I}-\dot{z}_{I}\right)$,

from which, using Eq. (13), we have the following electromagnetic force

$$
\hat{F}_{I}=\frac{\left(B_{I} l_{I}\right)^{2}}{\hat{R}_{I}+r_{I}}\left(\dot{\tilde{Z}}_{I}-\dot{\tilde{z}}_{I}\right)=C_{I}\left(\dot{\tilde{Z}}_{I}-\dot{\tilde{z}}_{I}\right), C_{I}=\frac{\left(B_{I} l_{I}\right)^{2}}{\hat{R}_{I}+r_{I}}
$$


As used in Eq. (9), $C_{I}$ is called the damping coefficient of the energy converter, which is determined by the parameters of the electromagnetic suspension unit. The collected power of the energy converter $I$ is calculated by

$$
\begin{aligned}
\hat{P}_{I} & =\hat{I}_{I}^{2} \hat{R}_{I}=\frac{C_{I} \hat{R}_{I}}{R_{I}}\left(\dot{Z}_{I}-\dot{z}_{I}\right)^{2} \\
& =\frac{C_{I} \hat{R}_{I}}{R_{I}}\left[\begin{array}{ll}
\dot{Z}_{I} & \dot{z}_{I}
\end{array}\right]\left[\begin{array}{cc}
1 & -1 \\
-1 & 1
\end{array}\right]\left[\begin{array}{l}
\dot{Z}_{I} \\
\dot{z}_{I}
\end{array}\right], \quad \hat{P}=\sum_{I=1}^{4} \hat{P}_{I},
\end{aligned}
$$

of which the percentages of energy collection with the corresponding time averaged values are respectively given by

$$
\begin{gathered}
\lambda_{I}=\hat{P}_{I} / P_{I}, \quad \bar{\lambda}_{I}=\left\langle\hat{P}_{I}\right\rangle /\left\langle P_{I}\right\rangle, \\
\lambda=\hat{P} / P, \quad \bar{\lambda}=\langle\hat{P}\rangle /\langle P\rangle .
\end{gathered}
$$

\subsection{Road uneven functions}

The uneven road is described by the following function with unit amplitude

$h(x, y),|h(x, y)| \leq 1$,

and this function may be considered as a non-dimensional shape function, of which the positive value implies a convex road while the negative value denotes a concave road. Since the system is considered as linear system, if the amplitude of the uneven road is not a unit, such as $H$, the dynamic response and the power can be obtained by multiplying them by $H$ and $H^{2}$, respectively. Considering our assumption of the vehicle moving along $o-x$ direction in the constant speed $V$ and the distance between the front and rear wheels, we can represent the road functions for each wheel as follows

$h_{I}= \begin{cases}h_{I}\left(V t, y_{I}\right), & \text { front: } I=1,2, \\ h_{I}\left(V t-a-b, y_{I}\right), & \text { rear: } I=3,4,\end{cases}$

in which the time when the front wheels reach the uneven road surface, is taken as the initial time $t=0$ in our analysis.

\subsection{Vibration isolation factor vectors}

To study the performance of the proposed energy harvesting device for reducing the vehicle vibrations due to uneven road excitations, we define the following vibration isolation factors. Based on Eq. (9), we consider the motions of the vehicle body caused by a unit maximum road height, so that we define the amplifying factor vectors

$\boldsymbol{\alpha}_{d}=\left[\begin{array}{lll}Z & \mathbf{Z}_{s}^{\mathrm{T}}\end{array}\right]^{\mathrm{T}}, \quad \boldsymbol{\alpha}_{v}=\left[\begin{array}{lll}\dot{Z} & \dot{\boldsymbol{Z}}_{s}^{\mathrm{T}}\end{array}\right]^{\mathrm{T}}, \quad \boldsymbol{\alpha}_{a}=\left[\ddot{Z}_{Z} \ddot{\boldsymbol{Z}}_{s}^{\mathrm{T}}\right]^{\mathrm{T}}$.
These vectors give the vibration levels of displacement, velocity, and acceleration at the vehicle mass center and the four points on the vehicle body at which the four energy converters are connected.

\section{Solution approach}

\subsection{Natural vibration}

To use the mode summation method to solve Eq. (9), we need to solve its corresponding natural vibrations governed by Eq. (9) with no damping $(\boldsymbol{C}=0)$ and no external force $(\boldsymbol{F}=0)$, i.e.,

$M \ddot{Z}+K Z=\mathbf{0}$.

We consider a solution of this equation in the form

$Z=\varphi \mathrm{e}^{\mathrm{j} \Omega t}$,

which, when substituted into Eq. (23), gives

$\left(-\Omega^{2} \boldsymbol{M}+\boldsymbol{K}\right) \boldsymbol{\varphi}=\mathbf{0}$.

The condition for a non-zero solution of Eq. (25) requires its determinant of the coefficient matrix to vanish, which gives the characteristic equation

$\left|\left(-\Omega^{2} \boldsymbol{M}+\boldsymbol{K}\right)\right|=0$.

The solutions of Eq. (26) are the seven natural frequencies $\Omega_{i}(i=1,2, \ldots, 7)$, of the system, which is represented by a diagonal matrix

$\boldsymbol{\Lambda}=\operatorname{diag}\left(\Omega_{i}\right)$.

Substituting the natural frequency $\Omega_{i}$ into Eq. (25), we obtain the corresponding natural mode vector $\varphi_{i}$ satisfying the orthogonal relationships

$$
\boldsymbol{\Phi}^{\mathrm{T}} \boldsymbol{M} \boldsymbol{\Phi}=\boldsymbol{I}, \quad \boldsymbol{\Phi}^{\mathrm{T}} \boldsymbol{K} \boldsymbol{\Phi}=\boldsymbol{\Lambda}^{2}, \quad \boldsymbol{\Phi}=\left[\boldsymbol{\varphi}_{1} \boldsymbol{\varphi}_{2} \cdots \boldsymbol{\varphi}_{7}\right] .
$$

\subsection{Mode summation}

Using the mode summation method, we represent the vehicle displacement vector

$Z=\Phi q$,

where $\boldsymbol{q}$, a time function, is called as the generalized coordinate vector of the mode space. Substituting Eq. (29) into Eq. (9), and then pre-multiplying the resultant equation by 
$\boldsymbol{\Phi}^{\mathrm{T}}$ as well as using the orthogonal condition Eq. (28), we obtain the mode equation

$\boldsymbol{I} \ddot{\boldsymbol{q}}+\boldsymbol{d} \dot{\boldsymbol{q}}+\boldsymbol{\Lambda}^{2} \boldsymbol{q}=\boldsymbol{f}, \quad \boldsymbol{d}=\boldsymbol{\Phi}^{\mathrm{T}} \boldsymbol{C} \boldsymbol{\Phi}, \quad \boldsymbol{f}=\boldsymbol{\Phi}^{\mathrm{T}} \boldsymbol{F}$.

Here, $\boldsymbol{f}$ is called as the generalized force vector and $\boldsymbol{d}$ is the damping matrix in the mode coordinate space. This damping matrix is not a diagonal matrix in general cases.

To judge the efficiency of energy collection and vibration isolation provided by the energy converters, we assume that the uneven road provides a harmonic excitation of unit amplitude and frequency $\tilde{\omega}$, represented by a complex force $\breve{f}=\breve{F} \mathrm{e}^{\mathrm{j} \tilde{\omega} \tilde{t}}$, of which its imaginary part denotes the real force, i.e.,

$\boldsymbol{f}=\operatorname{Im}\{\breve{f}\}=\operatorname{Im}\left\{\breve{\boldsymbol{F}} \mathrm{e}^{\mathrm{j} \tilde{\omega} \tilde{t}}\right\}$.

In this representation form, the dynamic response of the system can also be represented in a complex form

$\boldsymbol{q}=\operatorname{Im}\{\breve{q}\}, \quad \breve{\boldsymbol{q}}=\breve{\boldsymbol{Q}} \mathrm{e}^{\mathrm{j} \tilde{\omega} \tilde{t}}$,

which transforms Eq. (30) into a corresponding complex form

$\left(\boldsymbol{\Lambda}^{2}-\tilde{\omega}^{2} \boldsymbol{I}+\mathrm{j} \tilde{\omega} \boldsymbol{d}\right) \breve{\boldsymbol{Q}}=\breve{\boldsymbol{F}}$.

Pre-multiplying this equation by $\boldsymbol{\Lambda}^{-2}$, we obtain a nondimensional equation

$$
\begin{aligned}
& \left(\boldsymbol{I}-\eta^{2}+2 \mathrm{j} \eta \zeta\right) \breve{\boldsymbol{Q}}=\widehat{\boldsymbol{F}}, \quad \widehat{\boldsymbol{F}}=\boldsymbol{\Lambda}^{-2} \breve{\boldsymbol{F}}, \\
& \boldsymbol{\eta}=\operatorname{diag}\left(\omega / \Omega_{i}\right), \quad \zeta=\boldsymbol{\Lambda}^{-1} \boldsymbol{d} / 2 .
\end{aligned}
$$

Here, $\boldsymbol{\eta}$ is a non-dimensional diagonal matrix of frequency ratio and $\zeta$ is a non-dimensional mode damping matrix. From this equation, we obtain the complex amplitude of the generalized coordinate $\breve{q}$ in the form

$\breve{\boldsymbol{Q}}=\left(\boldsymbol{I}-\boldsymbol{\eta}^{2}+2 \mathrm{j} \eta \zeta\right)^{-1} \widehat{\boldsymbol{F}}$,

which, when substituted into Eq. (29), gives the complex/real physical displacement vector

$\breve{Z}=\boldsymbol{\Phi} \breve{Q} \mathrm{e}^{\mathrm{j} \tilde{\omega} \tilde{t}}, \quad \tilde{\boldsymbol{Z}}=\operatorname{Im}\{\breve{Z}\}$.

It should be mentioned herein, we use the complex representations to solve Eq. (9), of which the solutions are complex and their corresponding imaginary parts are the physical variables. As indicated in the book [38], when calculating powers by Eqs. (10) and (18), you have to use the real physical variables to get their correct results.

\section{Case study}

\subsection{Vehicle parameters}

We use the vehicle parameters given in Ref. [41]: sprung mass $\bar{M}=1440 \mathrm{~kg}$ with its roll inertia $J_{X}=900 \mathrm{~kg} \cdot \mathrm{m}^{2}$ and pitch inertia $J_{Y}=2000 \mathrm{~kg} \cdot \mathrm{m}^{2}$; distance of mass center from front axle $a=1.016 \mathrm{~m}$ and from rear axle $b=1.524 \mathrm{~m}$; front/rear track width $d=1.5 \mathrm{~m}$; front suspension stiffness $K_{I}=35,000 \mathrm{~N} \cdot \mathrm{m}^{-1}$ with energy harvester damping coefficient $C_{I}=2500 \mathrm{~N} \cdot \mathrm{s} \cdot \mathrm{m}^{-1}(I=1,2)$; rear suspension stiffness $K_{I}=30,000 \mathrm{~N} \cdot \mathrm{m}^{-1}$ with damping coefficient $C_{I}=2000 \mathrm{~N} \cdot \mathrm{s} \cdot \mathrm{m}^{-1}(I=3,4)$; front/rear un-sprung mass $m_{I}=80 \mathrm{~kg}$ with tire stiffness $k_{I}=200,000 \mathrm{~N} \cdot \mathrm{m}^{-1}$ and damping $c_{I}=10 \mathrm{~N} \cdot \mathrm{s} \cdot \mathrm{m}^{-1}(I=1,2,3,4)$. These parameters and their geometrical layout shown in Fig. 1, when substituted into Eq. (3), give the related prescribed matrices in Eq. (9), such as

$\boldsymbol{X}_{S}=\left[\begin{array}{llll}-d / 2 & d / 2 & -d / 2 & d / 2 \\ -a & -a & b & b\end{array}\right]$.

\subsection{Road functions}

We use the maximum height of the uneven road to measure the length, so that the road function will provide a non-dimensional unit height road excitation. You can assume any types of road functions, continues or discrete, to use the developed model for the corresponding simulation. Here, for an illustration purpose, we choose the following two road functions.

\section{Sinusoidal road function}

A sinusoidal road function with unit amplitude is defined as

$h(x, y)=\sin \beta x$,

where $\beta$ is a non-dimensional parameter involving the spatial period of the road function. The big value of parameter $\beta$ corresponds a short spatial period of road function. For simplicity without losing the generality, we choose $\beta=1$ in the following derivations, so that Eq. (21) becomes

$$
h_{I}= \begin{cases}\sin (\beta V t)=\sin (V t), & \text { front wheels: } I=1,2, \\ \sin [\beta(V t-a-b,)]=\sin (V t-a-b,), & \text { rear wheels: } I=3,4\end{cases}
$$


of which the time derivative

$\dot{h}_{I}= \begin{cases}\beta V \cos (V t)=V \cos (V t), & \text { front wheels: } I=1,2, \\ \beta V \cos [\beta(V t-a-b,)]=V \cos (V t-a-b,), & \text { back wheels: } I=3,4 .\end{cases}$

Therefore, from Eq. (9) we find that the vehicle moving speed $V$ involves a road excitation frequency, i.e.,

$\omega=\beta V=V$.

In this equation, the vehicle speed is measured by the road amplitude, so that its physical dimension, $\mathrm{m} \cdot \mathrm{s}^{-1} \cdot \mathrm{m}^{-1}=\mathrm{s}^{-1}$, is just the dimension of angular frequency $\omega$. The corresponding road vector of this road function is denoted by a complex vector as follows

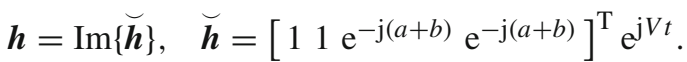

\section{A sine convex or concave road function}

To consider a type of ramp on roads, we assume a sine convex or concave road function defined in the range $0 \leq x \leq x_{0}$ in the form

$h= \begin{cases} \pm \sin x, & 0 \leq x<x_{0}, \\ 0, & x_{0} \leq x,\end{cases}$

$\dot{h}= \begin{cases} \pm V \cos x, & 0 \leq x<x_{0}, \\ 0, & x_{0} \leq x,\end{cases}$

$\ddot{h}= \begin{cases}\mp V^{2} \sin x, & 0 \leq x<x_{0}, \\ 0, & x_{0} \leq x .\end{cases}$

Therefore, for a single ramp in Eq. (42a), the road excitations applied to the four wheels take the form for the front wheels

$h_{I}= \begin{cases}\sin (V t), & 0 \leq t<x_{0} / V, \quad I=1,2, \\ 0, & x_{0} / V \leq t,\end{cases}$

and for the rear wheels

$h_{I}= \begin{cases}0, & 0 \leq t<(a+b) / V, \\ \sin (V t), & (a+b) / V \leq t<x_{0} / V, \quad I=3,4, \\ 0, & x_{0} / V \leq t .\end{cases}$

\subsection{Simulation results}

\subsubsection{Natural frequencies and modes}

The natural vibrations of the investigated system are governed by Eqs. (22)-(28), from which, when solving Eq. (25) for eigenvalues/eigenvectors, the following seven natural frequencies with the corresponding modes are obtained as shown in Fig. 2. Table 1 gives the detailed information of frequencies and mode shape characteristics of these seven natural modes. For each mode shape in Fig. 2, the left side is the front of vehicle, the dashed line denotes the stationary position of the vehicle while the real line represents the mode shape displacement, i.e., shift from the dashed position. The first three modes are observed with very small motions of the energy harvesters, while the last four modes are with the large energy harvester motions. The mode 1 of frequency $1.3274 \mathrm{~Hz}$ shows a rolling mode of the vehicle while the modes 2 and 3 represent two pitch modes of the vehicle in which mode 2 of frequency $1.3424 \mathrm{~Hz}$ and mode 3 of frequency $1.5682 \mathrm{~Hz}$ relate to the large motion amplitudes at the vehicle rear and front sides, respectively. Mode 4 of frequency $8.5404 \mathrm{~Hz}$ and mode 5 of frequency $8.556 \mathrm{~Hz}$ show respectively that the harvester masses 3 and 4, at the vehicle rear side, undergo the rolling and heave motions, while mode 6 of $8.6366 \mathrm{~Hz}$ and mode 7 of $8.6450 \mathrm{~Hz}$ behave the opposite motion shapes of the harvester masses 1 and 2, connected to the vehicle front side, with the rolling and heave motions.

In the energy harvesting suspension unit designs for vehicles to isolate the vibrations caused by road excitations as well as to collect this vibration energy, the following general rules involving natural modes may be followed.

(1) The energy collected from the energy harvester is proportional to the square of the relative velocity between the vehicle and harvester mass motions, as given by Eq. (18), so that the modes with large relative velocities will provide the large contribution for energy collection and vibration reduction.

(2) In principle, considering the symmetry of the system, for symmetrical road excitations, the dynamic responses of the anti-symmetrical modes vanish, therefore, they will not provide any contributions for energy collection and vibration isolation caused by the symmetrical road excitations. Similarly, for anti-symmetrical road excitations, symmetrical modes have also no contributions for harvesting energy with vibration isolation. As shown in Fig. 2 and Table 1, the modes 2, 3, 5, 7 provide symmetric motions about the central line of the vehicle, while the modes $1,4,6$ are antisymmetrical. 

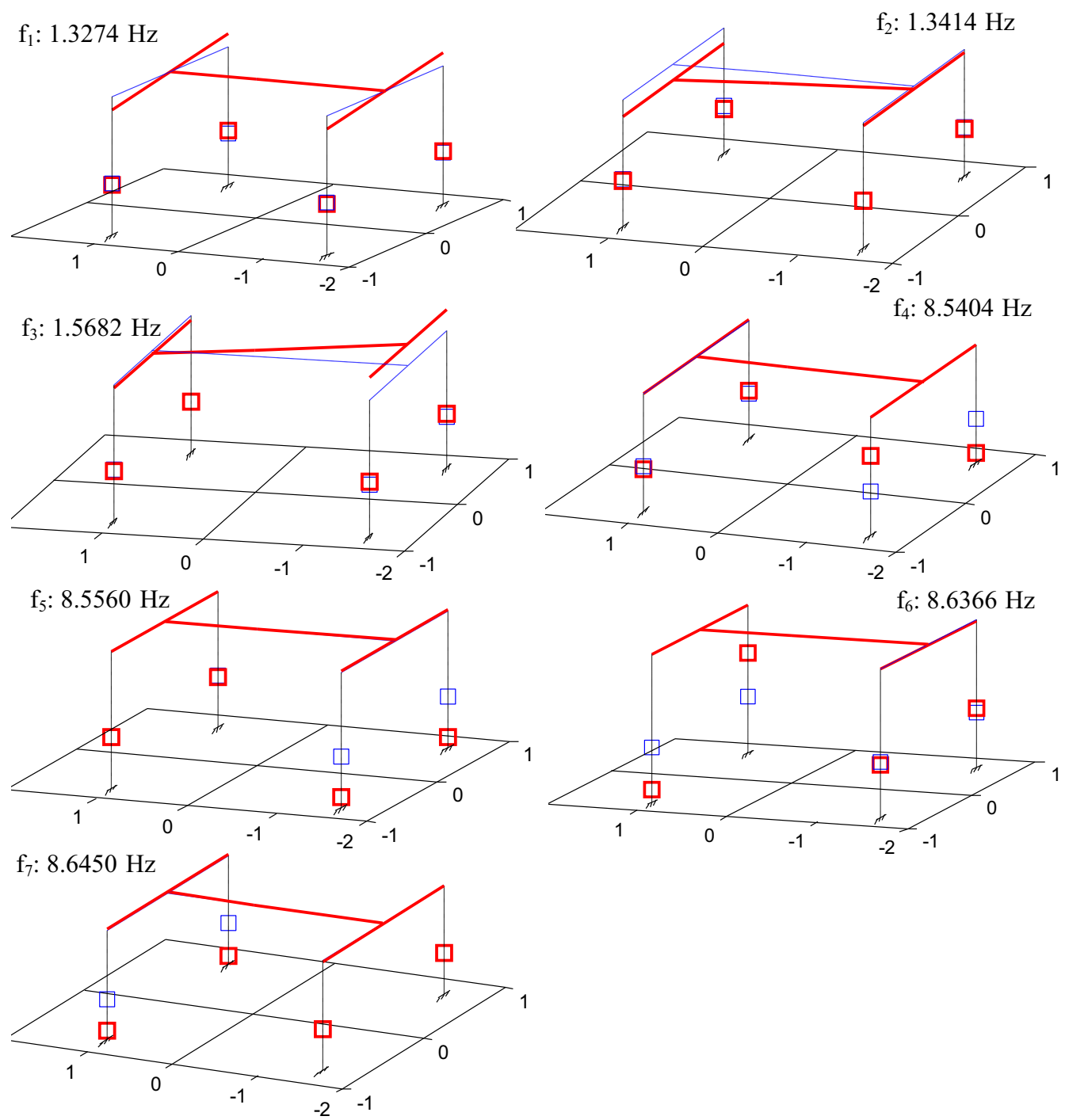

Fig. 2 Seven natural mode shapes of the integrated vehicle-energy harvester-tire system, of which for each, its left side is the front of vehicle, and the dashed line denotes the stationary vehicle position while the real line shows the mode shift motion from the dashed position

\subsubsection{Energy collection and vibration isolation}

Based on the mode summation approach and choosing different road functions with the unit amplitude as given by Eq. (41) or (42), we solve the dynamic equations of the system to investigate the performance of energy collections and vibration isolations of the proposed electromagnetic energy harvester for cases 1, 2, 3, 4, respectively with the energy harvester damping coefficients $25 \%, 50 \%, 75 \%$, and $100 \% C_{I}$. The simulation results are given in the frequency response spectrums or the time histories as follows.

\section{Symmetrical continuous sinusoidal road excitation}

For a continuous sinusoidal road excitation defined by Eq. (41), where the vehicle speed links the angular frequency of the road excitation. After solving the dynamic equa- tions subject to this road excitation, we obtain the following frequency response spectrums of displacements, velocities, accelerations at chosen points of the system as well as the road input energy and the harvested energy for the four cases with different energy harvester damping coefficients. In these figures, the horizontal axes denote the road excitation frequency $\omega=V$, i.e., the vehicle speed, while the vertical axes represent the related dynamic response variables excited by a unit amplitude $(\mathrm{m})$ road excitation. These curves provide the base to examine the performance of the energy harvester suspension system in reducing vibrations and harvesting energy.

Figure 3 shows the response curves of vertical displacement $Z$, velocity, and acceleration at the mass center and the roll/pitch angles $\theta_{x}$ and $\theta_{y}$ with the corresponding angular velocities and angular accelerations of the vehicle, which describes the integrated motion of rigid vehicle. The energy 
Table 1 The frequencies and mode shape characteristics of seven natural modes of integrated vehicle-energy harvester-tire interaction system

\begin{tabular}{lllll}
\hline Mode number & $\begin{array}{l}\text { Frequency } \\
(\mathrm{Hz})\end{array}$ & $\begin{array}{l}\text { Angular } \\
\text { frequency (rad/s) }\end{array}$ & $\begin{array}{l}\text { Symmetry (S) } \\
\text { or anti-symmetry } \\
(\text { AS) }\end{array}$ & $\begin{array}{l}\text { Vehicle (V) } \\
\text { /E-converter } \\
\text { motion }\end{array}$ \\
\hline 1 & 1.3274 & AS & V. Roll \\
2 & 1.3414 & 8.3401 & $\mathrm{~S}$ & V. Rear pitch \\
3 & 1.5682 & 8.4280 & $\mathrm{~S}$ & V. Front pitch \\
4 & 8.5404 & 9.8530 & AS & E. Rear roll \\
5 & 8.5560 & 53.659 & $\mathrm{~S}$ & E. Rear pitch \\
6 & 8.6366 & 53.757 & AS & E. Front roll \\
7 & 8.6450 & 54.267 & $\mathrm{~S}$ & E. Front pitch \\
\hline
\end{tabular}

collection of each harvester involves the relative motion between the vehicle and the harvester body, so that as an example, Fig. 4 presents the dynamic response spectrums of the displacement $z_{1}$, velocity $\dot{z}_{1}$, and acceleration $\ddot{z}_{1}$ at the mass $m_{1}$ of energy converter 1 , the corresponding ones $Z_{1}$, $\dot{Z}_{1}, \ddot{Z}_{1}$ at vehicle point 1 , where converter 1 fixed, as well as their differences $z_{1}-Z_{1}, \dot{z}_{1}-\dot{Z}_{1}, \ddot{z}_{1}-\ddot{Z}_{1}$. Figure 6 gives the input power by the road excitation, the collection power by the energy harvester as well as the energy collection efficiency, in which for the lower frequency range the curves have been locally amplified to get its more details.

Observing these three Figs. 3-5, we can clearly see that the investigated symmetrical road excitation excites only symmetrical motions consisting of four symmetrical modes shown in Table 1 with the curve peaks located around 8-9 $\mathrm{rad} \cdot \mathrm{s}^{-1}$ and 53-54 $\mathrm{rad} \cdot \mathrm{s}^{-1}$. Especially, as shown in Fig. 4, the dynamic response of anti-symmetric rolling motion $\theta_{x}$ of the vehicle about $x$-axis is extremely small in the calculation error range. With increasing the energy harvester damping values, the vibration peaks of vehicle motions are much reduced, so that the power input due to vibrations and the harvesting energy are also reduced. Since in the simulations, we assume that the tire damping is quite small and neglect other types of physical damping, therefore, the nearly full vibration energy is collected. For practical cases, due to existence of various physical damping, a part of vibration energy will be dissipated, so that the energy collection efficiency is less than 1 .

Non-symmetrical continuous sinusoidal road excitation on the right side of vehicle

As discussed above, the symmetrical road excitation cannot excite the responses of the anti-symmetrical modes of the vehicle. Here, we consider a non-symmetrical continuous sinusoidal road excitation defined also by Eq. (41), but only acting on the right side wheels of the vehicle, i.e. the left side wheels of vehicle running along an even road with no road excitations. Figures 6-9 give the dynamic response spectrums in the similar structures of Figs. 3-5, respectively.
Since the road excitation is non-symmetrical, so that all modes of the system contribute to the dynamic responses. As shown in Fig. 6 for this excitation, the roll rotation motion $\theta_{x}, \dot{\theta}_{x}, \ddot{\theta}_{x}$, of the vehicle does not vanish. Furthermore, a comparison of the curves in Figs. 7 and 8 shows that the dynamic responses of the variables at positions 1 and 2 are obviously different for this case of the non-symmetrical road excitation applied only on the right side of vehicle. The response values on the left side position 2 shown in Fig. 8 are much smaller than the ones on the right side position 1 as given in Fig. 7.

For this non-symmetrical continuous sinusoidal road excitation, we consider the road excitation of amplitude $0.2 \mathrm{~m}$ and use the standard Newmark time integration method [42] with time step $\Delta t=0.0005 \mathrm{~s}$ in the simulations to reveal the characteristics of time histories of the integrated system, which are shown in Figs. 10 and 11. Figure 10 shows the time histories of the displacements of vehicle points and suspension masses at positions 1 and 2 as well as the ones of the displacement at vehicle mass center and its roll/pitch rotation angles, while Fig. 11 gives the time histories of input and collection powers. It is observed that:

(1) The displacement at excitation side 1 is larger than the non-excited side 2 .

(2) At the initial road impact instance, although the vehicle body suffers big motions but its order is much lower than the suspension mass motions, which absorbs the impact energy and reduces the road impact transmitting to the vehicle body.

(3) Since the system is linear, the stable response shows a sinusoidal form as a function of the frequency (vehicle speed).

(4) The input and output collection powers are positive, of which the values at vehicle speed 50 are maximum. This is because the speed $(\omega=50)$ is near to the resonance peak shown in Fig. 7, so that there is large relative motion $z_{1}-Z_{1}$ from which the large road excitation energy is collected. 

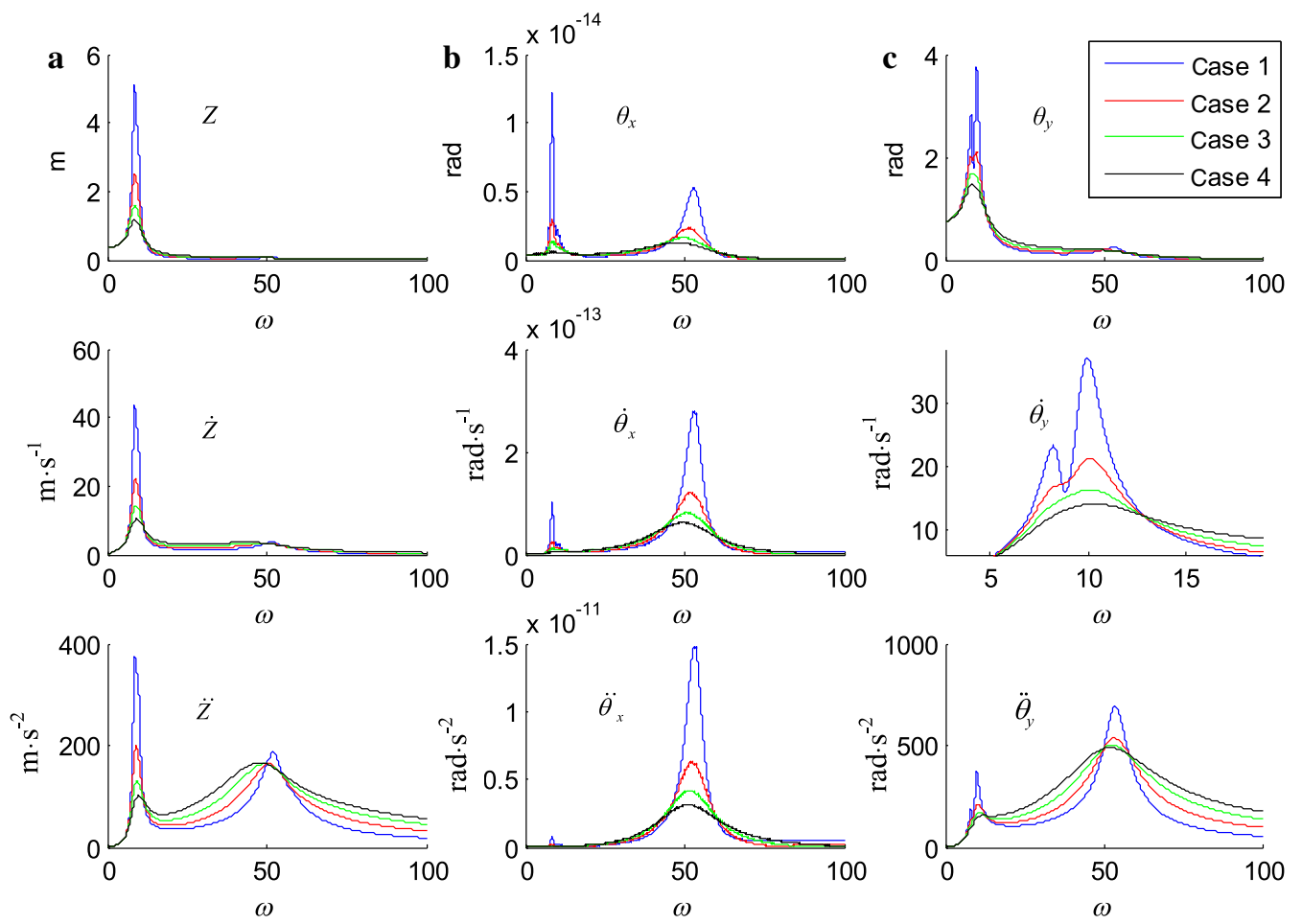

Fig. 3 (Color online) Frequency spectrums for displacement/rotation angles, their velocities and accelerations. a Displacement $Z, \dot{Z}$, and $\ddot{Z}$. b Rolling angle $\theta_{x}, \dot{\theta}_{x}$, and $\ddot{\theta}_{x}$. c Pitching angle $\theta_{y}, \dot{\theta}_{y}$, and $\ddot{\theta}_{y}$
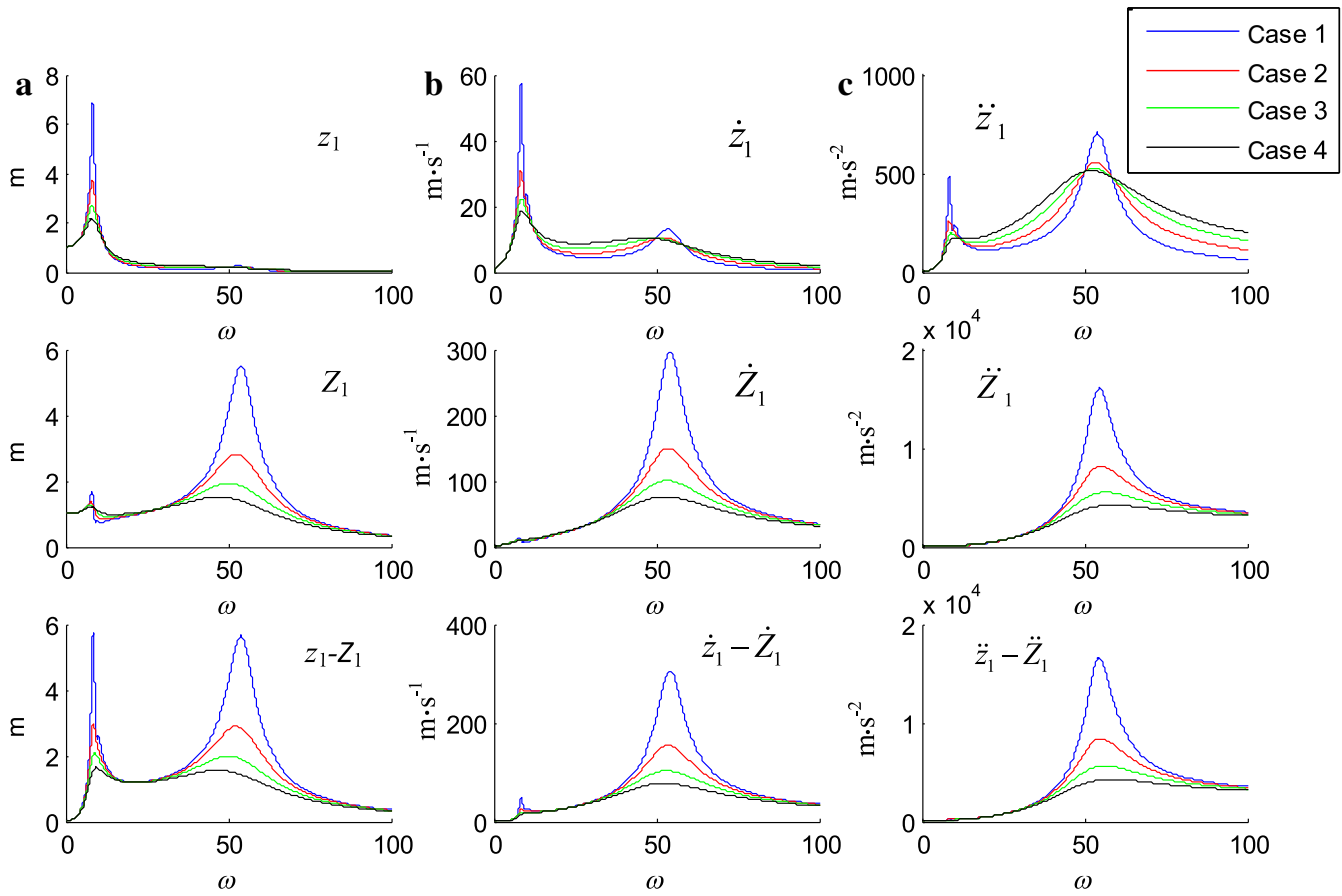

Fig. 4 (Color online) Dynamic response spectrums. a Displacements $z_{1}, Z_{1}$, and $z_{1}-Z_{1}$. b Velocities $\dot{z}_{1}, \dot{Z}_{1}$, and $\dot{z}_{1}-\dot{Z}_{1}$. c Accelerations $\ddot{z}_{1}, \ddot{Z}_{1}$, and $\ddot{z}_{1}-\ddot{Z}_{1}$

\section{A sine convex ramp}

Here, we consider the investigated vehicle, moving in different speeds $V=30,50,70 \mathrm{~m} \cdot \mathrm{s}^{-1}$, is impacted by a half sine convex ramp (amplitude $0.2 \mathrm{~m}, x_{0}=\pi$ in Eqs. $(42 \mathrm{~b}, 42 \mathrm{c})$ ) only at positions 1 and 3 on its right-side wheels in succession. Based on the standard Newmark approach [42], Fig. 12 gives the time histories of the displacements of four vehicle points $\left(Z_{1}-Z_{4}\right)$ and the 4 suspension masses points $\left(z_{1}-z_{4}\right)$ 

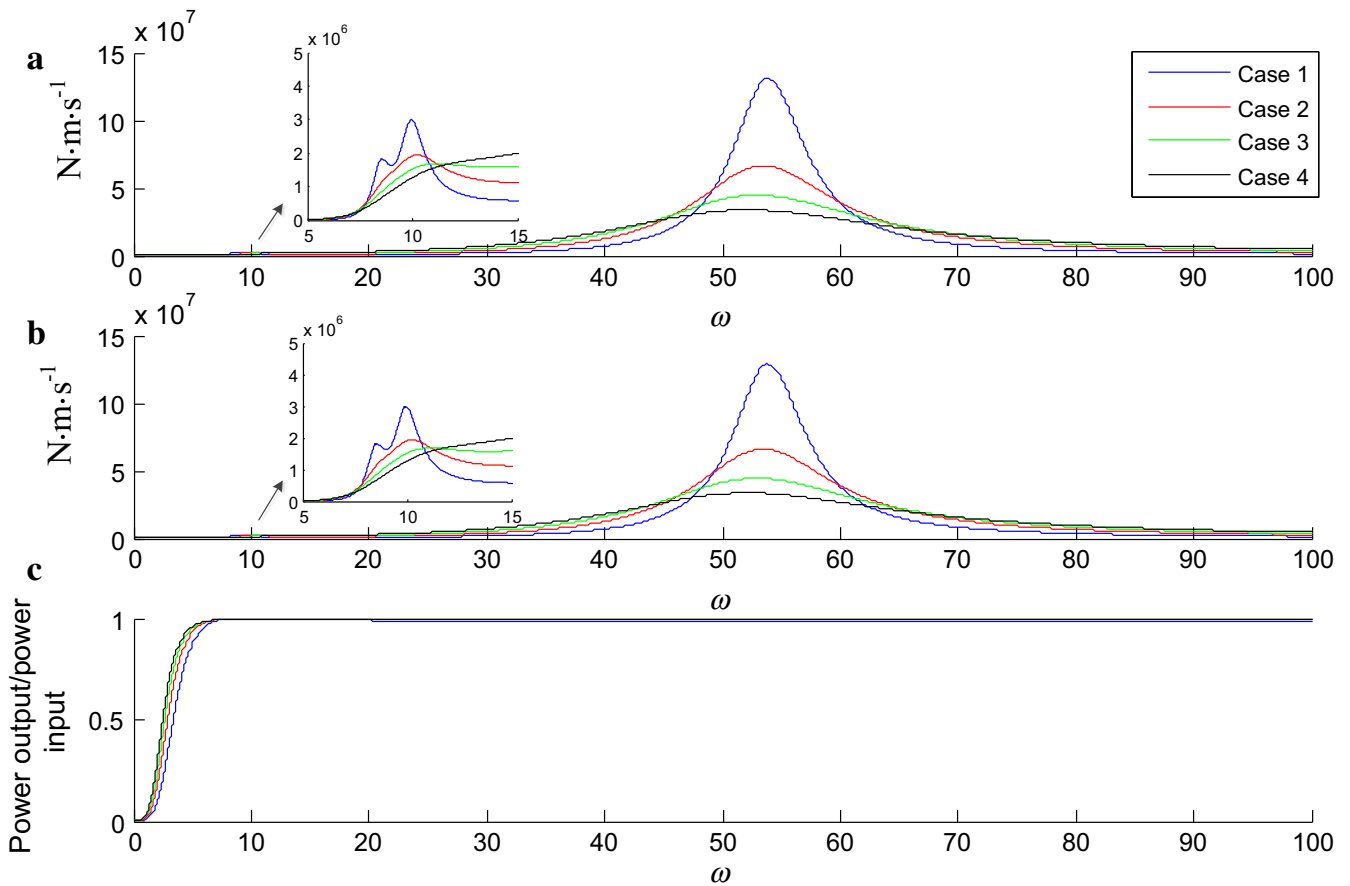

Fig. 5 (Color online) Frequency spectrums of powers. a Road excitation input. b Power collection. c Energy collection efficiency =collection power/input power
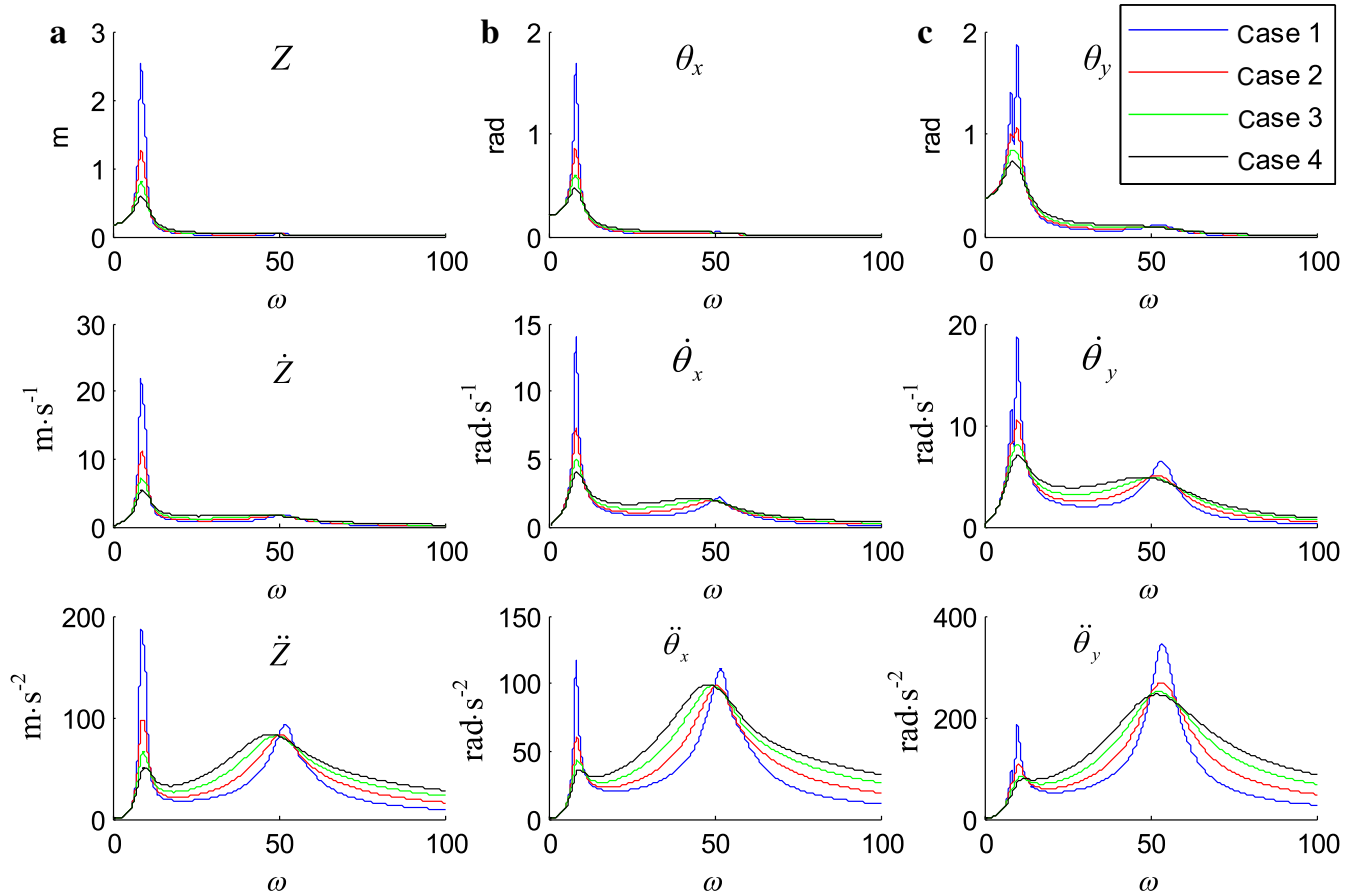

Fig. 6 (Color online) Frequency spectrums of displacement/rotation angles with their velocities and accelerations. a Displacement $Z, \dot{Z}$, and $\ddot{Z}$. b Rolling angle $\theta_{x}, \dot{\theta}_{x}$, and $\ddot{\theta}_{x}$. c Pitching angle $\theta_{y}, \dot{\theta}_{y}$, and $\ddot{\theta}_{y}$

while Fig. 13 shows the time histories of the displacement $Z$ at mass center and roll $\theta_{x} /$ pitch $\theta_{y}$ rotation angles of the vehicle. Figures 14 and 15 provide the instant time histories of road input powers and output collection powers at four sus- pension positions and the total ones of the integrated vehicle, respectively.

These time histories show that: 

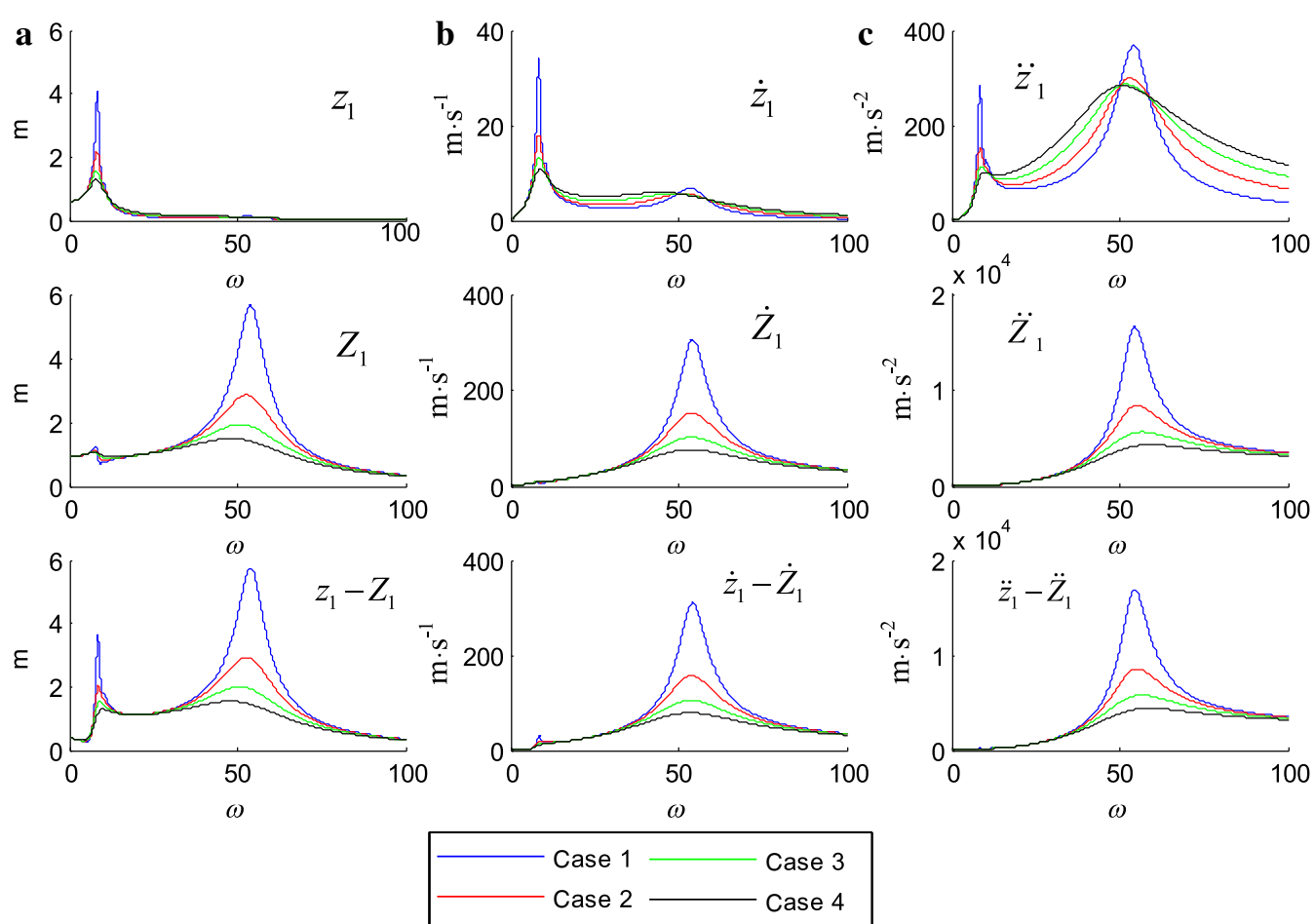

Fig. 7 (Color online) Dynamic response spectrums. a Displacements $z_{1}, Z_{1}$, and $z_{1}-Z_{1}$. b Velocities $\dot{z}_{1}, \dot{Z}_{1}$, and $\dot{z}_{1}-\dot{Z}_{1}$. c Accelerations $\ddot{z}_{1}$, $\ddot{Z}_{1}$, and $\ddot{z}_{1}-\ddot{Z}_{1}$
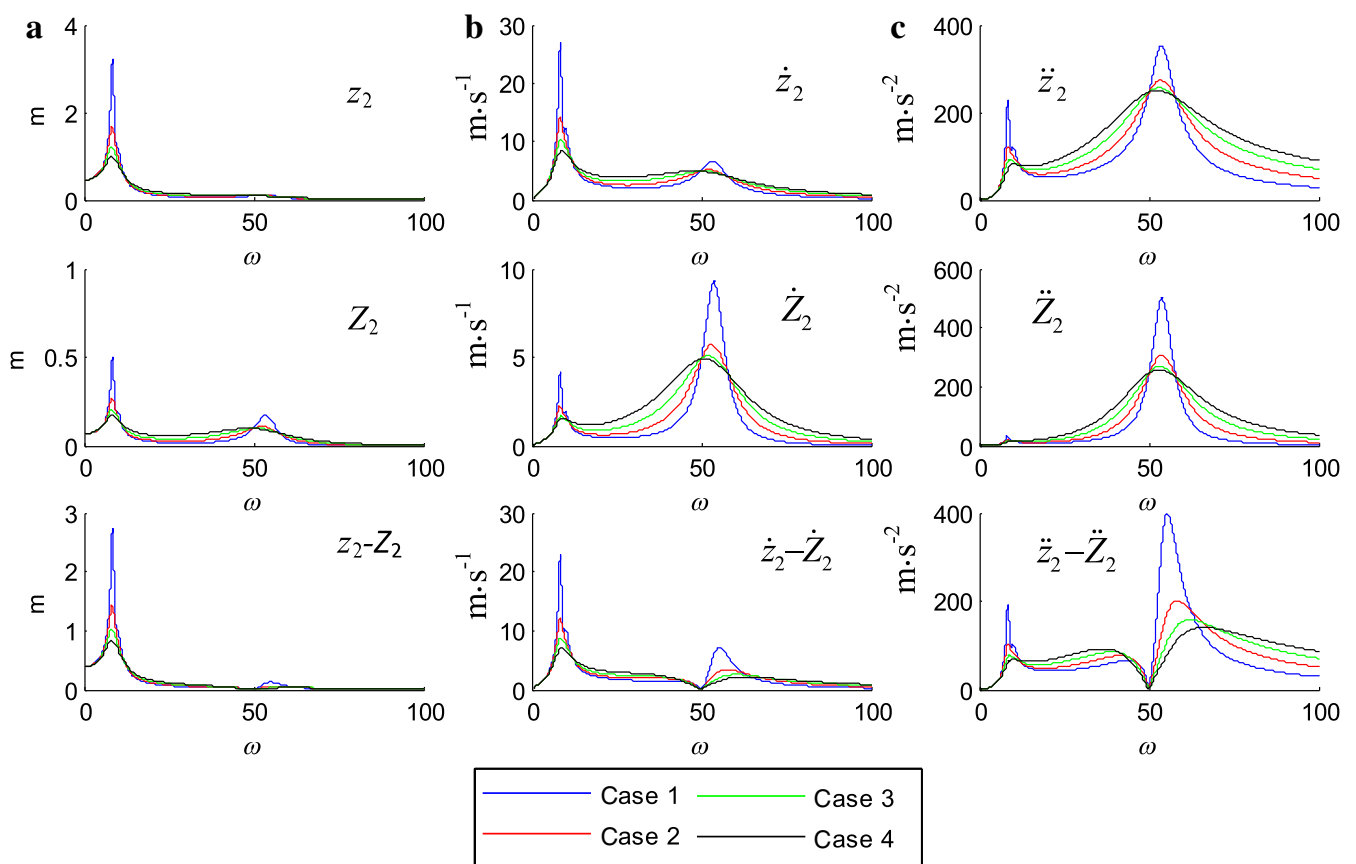

Case $2-$ Case 4

Fig. 8 (Color online) Dynamic response spectrums. a Displacements $z_{2}, Z_{2}$, and $z_{2}-Z_{2}$. b Velocities $\dot{z}_{2}, \dot{Z}_{2}$, and $\dot{z}_{2}-\dot{Z}_{2}$. c Accelerations $\ddot{z}_{2}$, $\ddot{Z}_{2}$, and $\ddot{z}_{2}-\ddot{Z}_{2}$

(1) The dynamic responses and the input/output powers of the system behave the transient impacting characteristics, of which at the time instants when the wheels impact by the road ramp, the curves show impact peaks. With the time elapsing, these impact peaks gradually tend to zero.
(2) The responses and the power collections at the impact positions 1 and 3 are much larger than the other two nonimpact positions 2 and 4, at which the input powers are zero as shown in Fig. 13 due to the even road surface. 


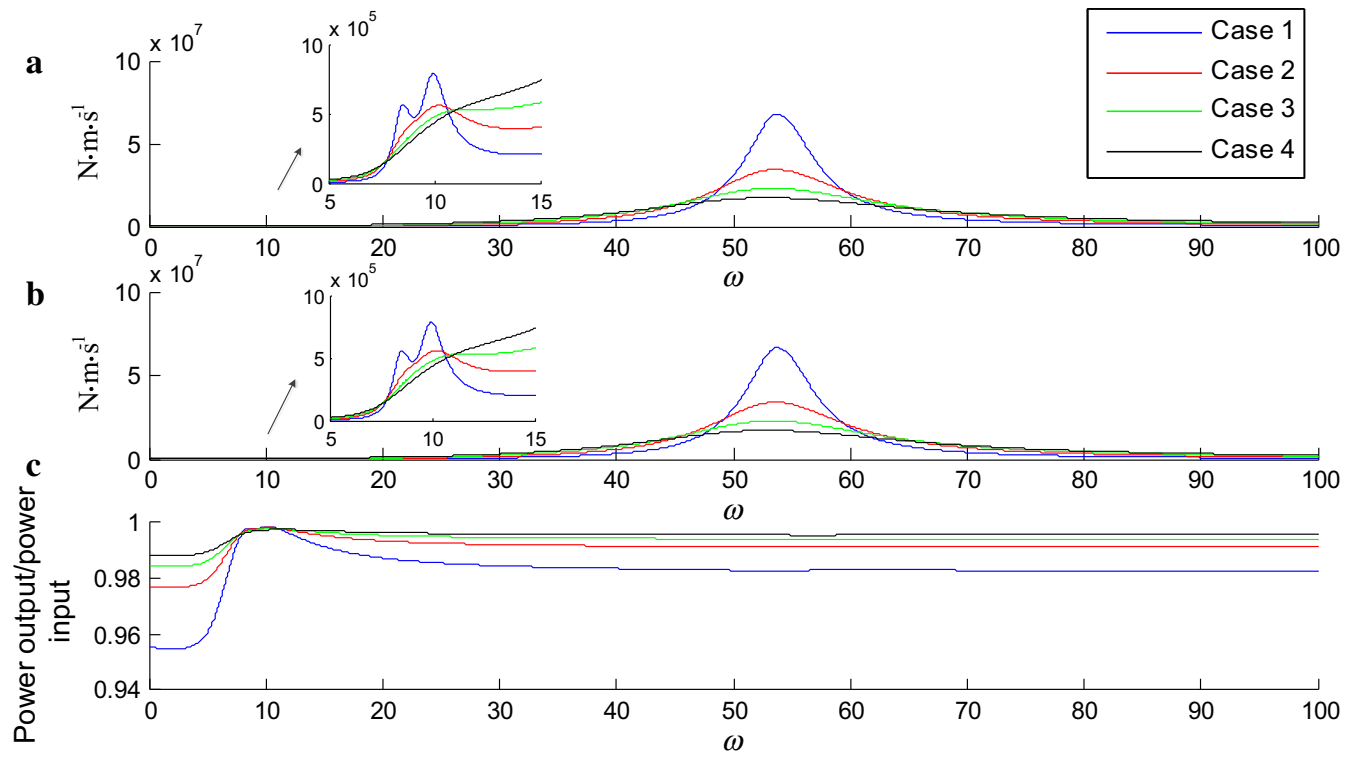

Fig. 9 (Color online) Frequency spectrums of powers. a Road excitation input. b Power collection. c Energy collection efficiency = collection power/input power
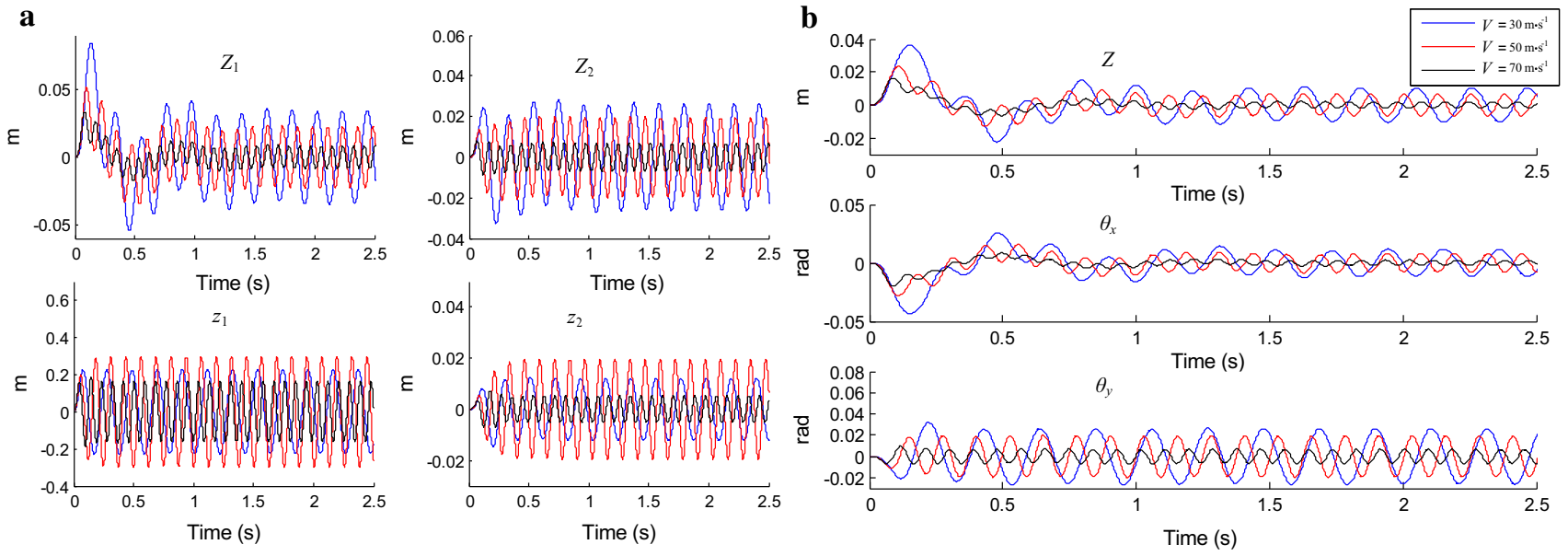

Fig. 10 (Color online) Time histories. a Displacements of vehicle points $\left(Z_{1}, Z_{2}\right)$ and suspension masses $\left(z_{1}, z_{2}\right)$ at positions 1 and 2 . b Displacement $(Z)$ at mass center, rolling $\left(\theta_{x}\right)$, and pitching $\left(\theta_{y}\right)$ rotation angles of the vehicle

(3) As shown in Fig. 14, until the time instant $(a+b) / V$, the rear wheel 3 gives no input power and small output power, since it is excited by the ramp with the time delay of $(a+b) / V$.

(4) As shown in Fig. 14, at vehicle speed $V=30 \mathrm{~m} \cdot \mathrm{s}^{-1}$, the non-impact positions 2 and 4 collect the large amount of energy, while at vehicle speed $V=70 \mathrm{~m} \cdot \mathrm{s}^{-1}$, the impact positions 1 and 3 collect the large amount of energy, which is unable to be reveal using a quarter vehicle model.

(5) The total energy collection increases with increasing vehicle speed as shown by Fig. 15, since the higher speed vehicle has a higher momentum which causes a higher impact resistance force provided by the vehicle engine with the higher energy output.

\section{Potential further research directions}

The following directions may be considered for future research.

(1) This paper has conducted research only on a fundamental concept but has not presented an idea on how practically to collect the harvested energy. The best possible way is to design an electrical device to transfer the collected energy into the battery of vehicle. However, since the electrical device operation needs power, a proper evaluation of final economic efficiency is required. Another option is to apply the collected energy to some vehicle facilities, such as window deforester, etc. To realize any 


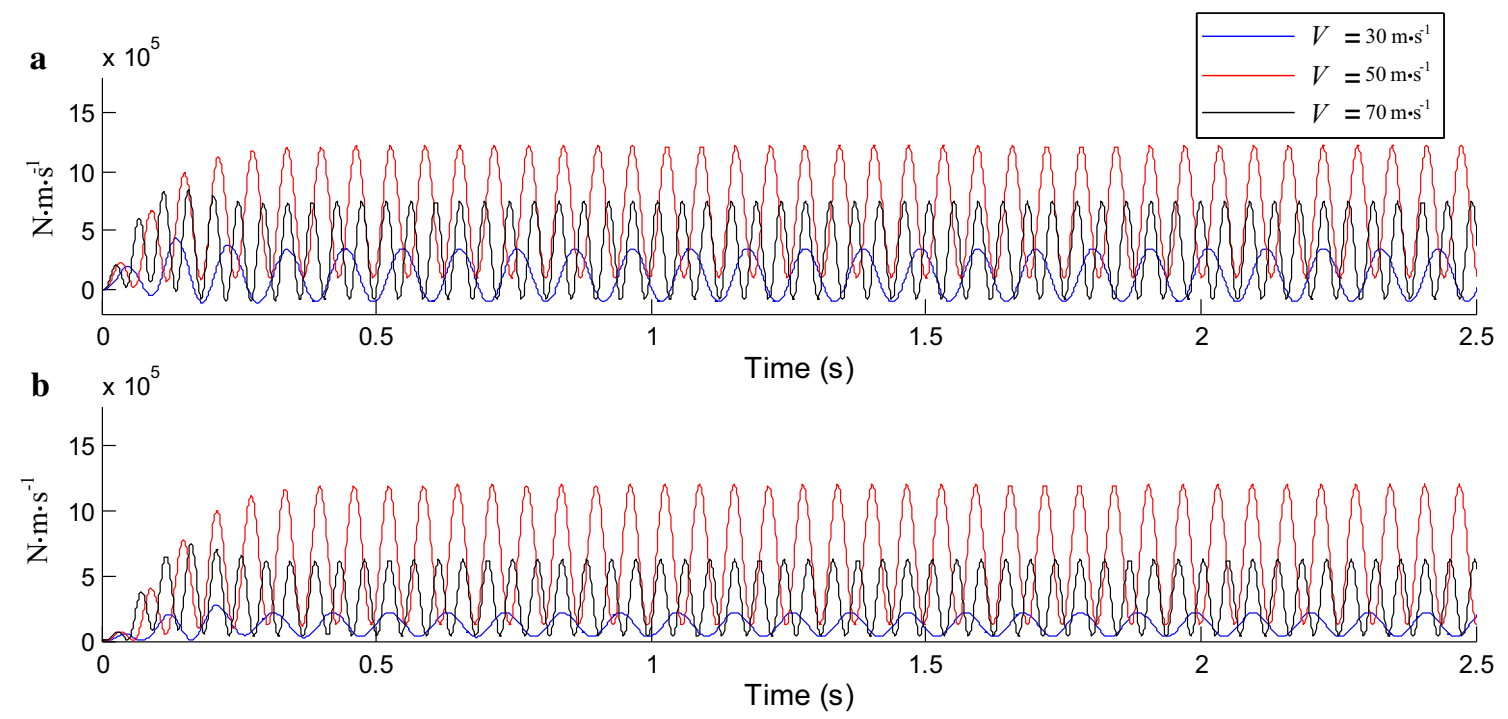

Fig. 11 (Color online) Time histories. a Road input power. b Output collection power
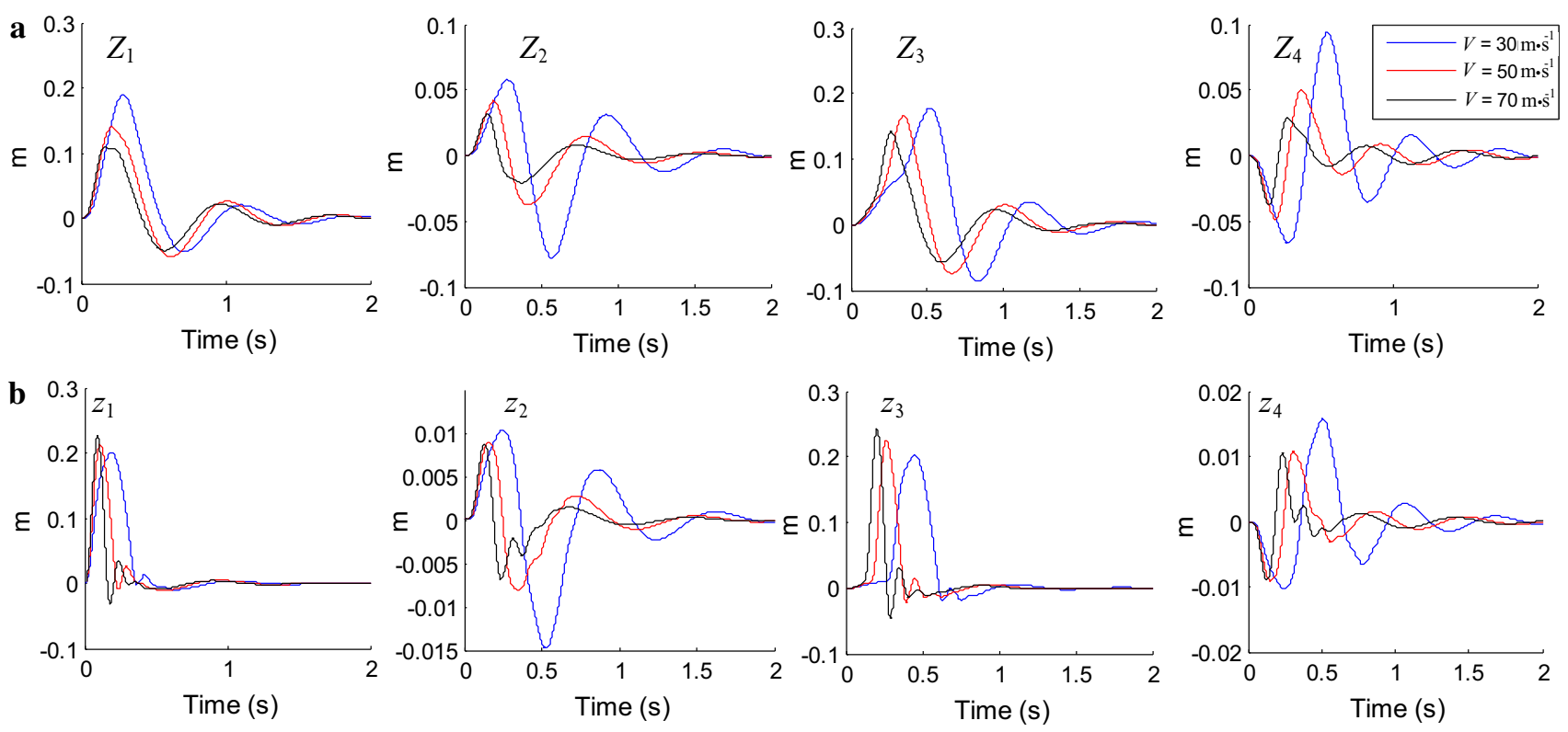

Fig. 12 (Color online) Time histories. a Displacements $\left(Z_{1}-Z_{4}\right)$ of four vehicle points. b Displacements $\left(z_{1}-z_{4}\right)$ at four suspension masses points

possible practical designs, analysis and tests are necessary.

(2) The other three motions of rigid vehicle, sway in $y$ direction, surge in $x$-direction and yaw about $z$-axis may be considered for a full rigid vehicle model. Especially, the sway and yaw motions are important for vehicle control performance.

(3) The further detailed research may consider the vehicle as an elastic body with more practical structure, for which finite element method may be used, but the solution approaches discussed in this paper are still valid.
The main difference of this case is the order of the final dynamic matrix equation is higher than 7 .

(4) The harvester damping depends on the parameters of electromagnetic body as well as its moving electric coil, therefore practical designs must be analyzed and tested to obtain the required damping value of energy harvester.

\section{Conclusions}

This paper has developed an integrated numerical model consisting of a rigid vehicle, four electromagnetic energy 


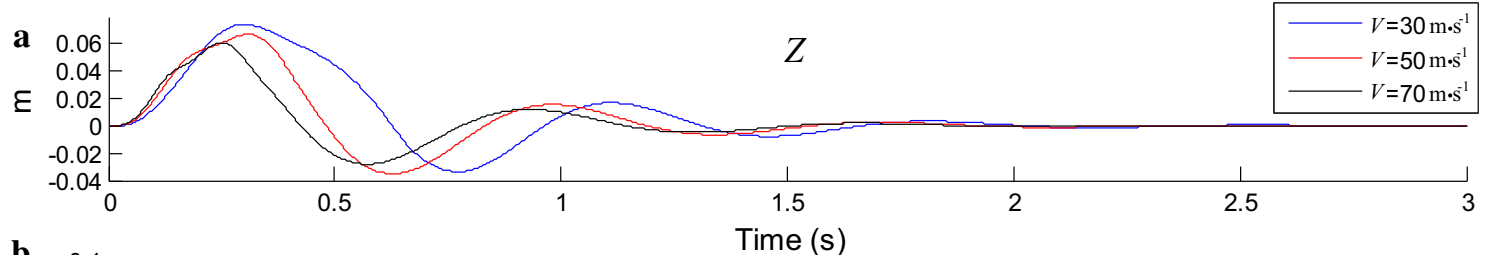

b

Time (s)

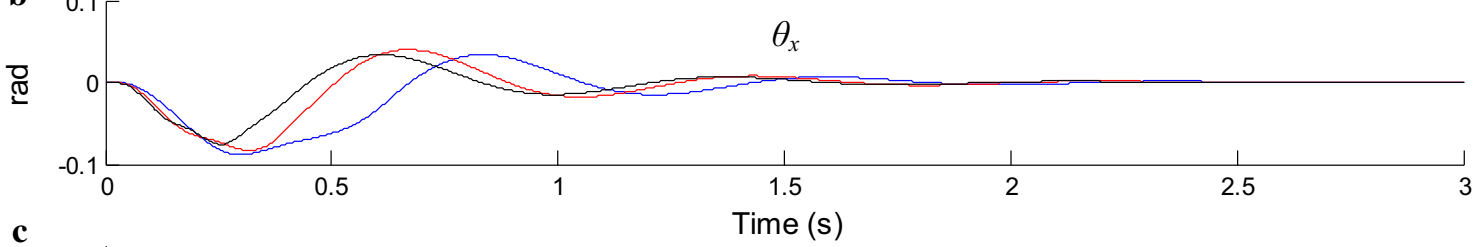

c

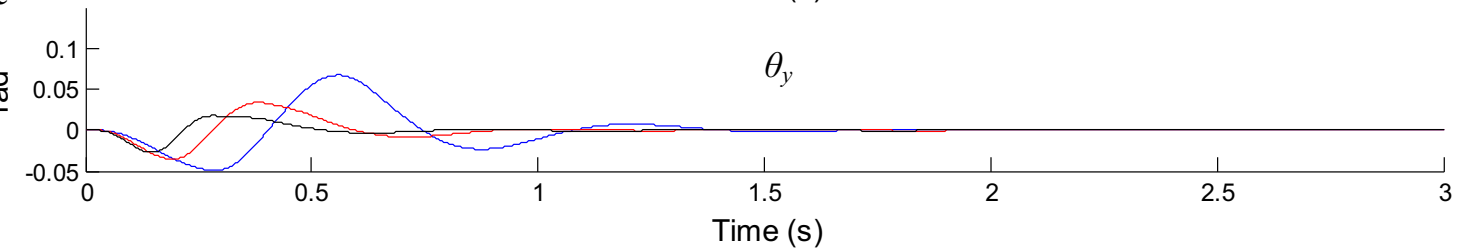

Fig. 13 (Color online) Time histories. a Displacement $Z$ at the mass center. b Rolling angle $\theta_{x}$. $\mathbf{c}$ Pitching angle $\theta_{y}$ of the vehicle
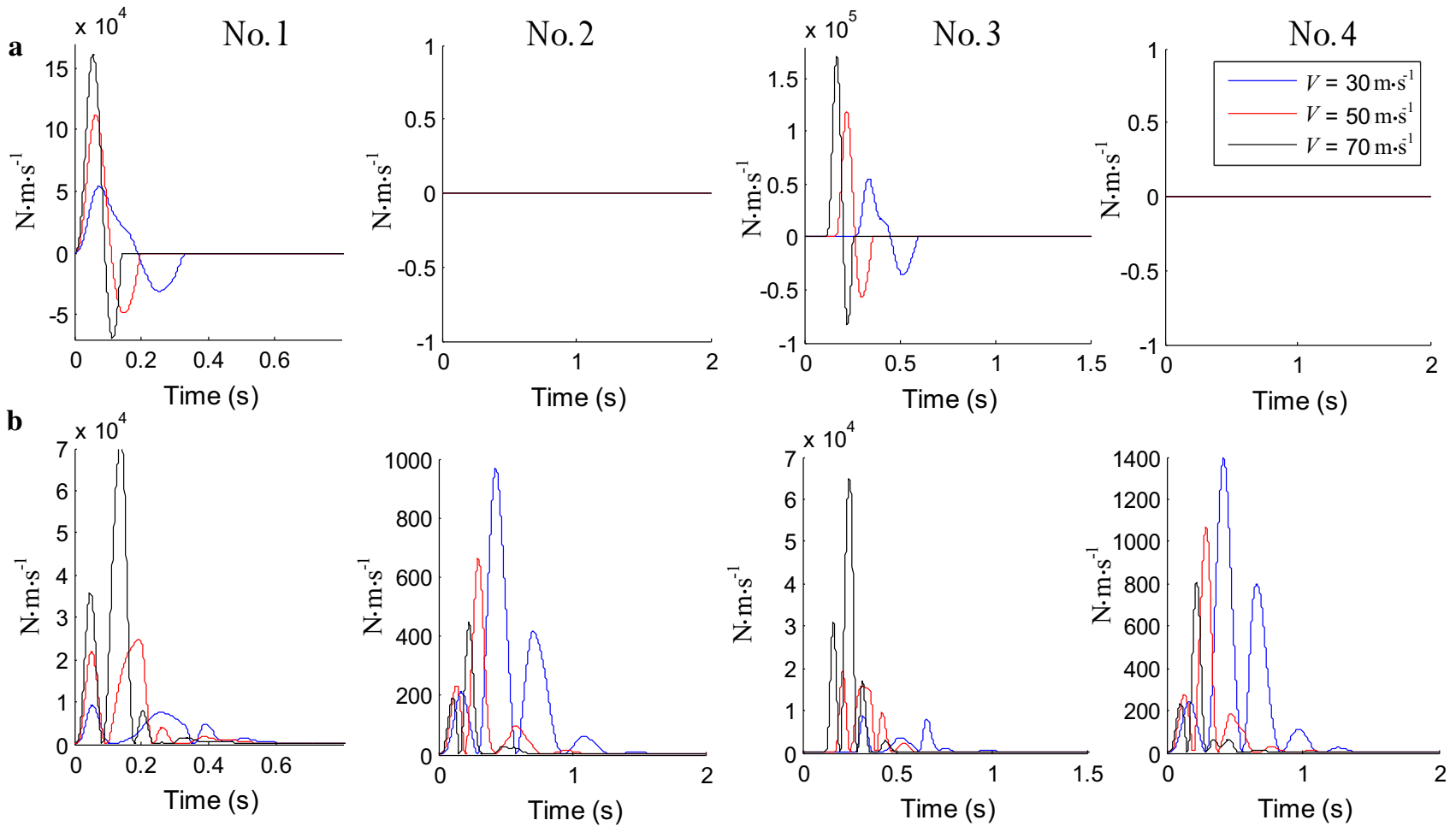

Fig. 14 (Color online) Time histories of powers. a Input powers at four wheels. b Collection powers at four electromagnetic energy harvesters

harvesters, and four vehicle tires to investigate and reveal the characteristics of the system in harvesting energy while reducing vibrations caused by uneven road excitations. The mathematical formulations with their solution approaches to the mechanical-electromagnetic interaction system subject to various uneven road excitations are presented. The theoretical analysis and numerical simulations demonstrate that this type of energy harvesting provides a damping mechanism in the integrated coupling system. The numerical examples for the continuous sine wave road excitation and the single ramp impact are simulated, from which the following guidelines are obtained: (1) the current passive vehicle suspension units can be replaced by the investigated electromagnetic energy harvesters in reducing vibrations 


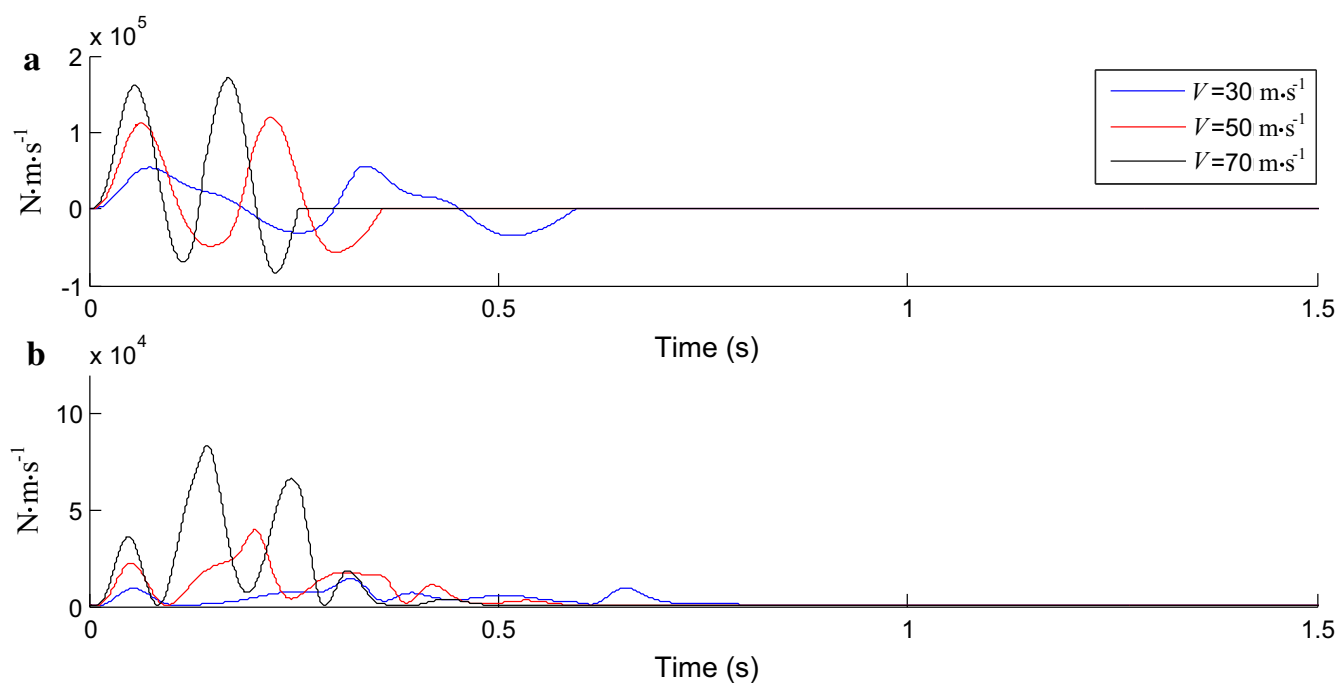

Fig. 15 (Color online) Time histories of powers. a Total road input power. b Total collection powers of the integrated vehicle

caused by uneven roads and at same time harvesting the lost engine energy; (2) the model with seven DOF reveals the more complex interaction characteristics of the system than a quarter vehicle model, so that it can address the efficiency of vibration isolation/energy harvesting affected by the natural modes/frequencies of the vehicle, the possible different road excitations on four wheels, vehicle speeds and road impact time delays for the front and rear wheels, etc. This model provides a means for vehicle electromagnetic suspension unit designs in engineering. Potential further research directions are suggested for readers to consider in future.

Acknowledgements The authors acknowledge CSC for supporting S. Zhou to visit University of Southampton for one year to engage in this research, as well as Harbin Engineering University for supporting J. T. Xing to visit Harbin Engineering University (Grant HEUCF160104).

Open Access This article is distributed under the terms of the Creative Commons Attribution 4.0 International License (http://creativecomm ons.org/licenses/by/4.0/), which permits unrestricted use, distribution, and reproduction in any medium, provided you give appropriate credit to the original author(s) and the source, provide a link to the Creative Commons license, and indicate if changes were made.

\section{References}

1. Thorpe, T.W.: A brief review of wave energy. A report produced for The UK Department of Trade and Industry. ETSU R-120, AEA Technology Plc. (1999)

2. Office of Naval Research: Environmental assessment for proposed wave energy technology project in Kaneohe Bay, Report by Office of Naval Research and US Department of the Navy, Hawaii. (2003)

3. Bedard, R., Hagerman, G., Previsic, M., et al: Offshore Wave Power Feasibility Demonstration Project: Final Summary ReportProject Definition Study, EPRI Global WP 009 - US Rev 1, The Electric Power Research Institute (2005)
4. Paasch, R., Ruehl, K., Howland, J., et al.: Wave energy: a pacific perspective. Philos. Trans. R. Soc. A 370, 481-501 (2012). doi:10. 1098/rsta.2011.0225

5. U.S. Department of the Interior: Technology white paper on wave energy potential on the U.S. Outer Continental Shelf. http:// ocsenergy.anl.gov (2006). (Accessed 3 Oct 2016)

6. Washio, Y.: Wave energy research and development at JAMSTEC, offshore floating wave energy device, Mighty Whale. http://www. takesteps.org/empower/exhibition (2006). (Accessed 3 Oct 2016)

7. Ingram, D.: Edinburgh makes waves in power generation research. http://www.ed.ac.uk/files/atoms/files/engineering-wavemakers. pdf (2016). (Accessed 3 Oct 2016)

8. Pelamis Wave Power: World's first commercial wave energy project, Agucadoura, Portugal. http://www.power-technology. com/projects/pelamis/ (2016). (Accessed 3 Oct 2016)

9. Wave Dragon: Wave Dragon has started the development of a 1.5 MW North Sea Demonstrator, March 2011. http://www. wavedragon.net (2016). (Accessed 3 Oct 2016)

10. Wave Plane Productions: Wave Plane-the only device in the world. http://www.waveplane.com (2016). (Accessed 3 Oct 2016)

11. Falnes, J.: Ocean Waves and Oscillating Systems, Linear Interactions Including Wave-Energy Extraction. Cambridge University Press, London (2002)

12. Xing, J.T., Xiong, Y.P., Tan, M., et al.: A numerical investigation of a wave energy harness device-water interaction system subject to the wave maker excitation in a towing tank. In: Proceedings of the ASME 28th International Conference on Ocean, Offshore and Arctic Engineering, Honolulu, Hawaii, 1-10(2009)

13. Xu, X., Pavlovskaia, E., Wiercigroch, M., et al.: Dynamic interactions between parametric pendulum and electro-dynamical shaker. Z. Angew. Math. Mech. 87, 172-186 (2007)

14. Yang, J., Xiong, Y.P., Xing, J.T.: Investigations on a nonlinear energy harvesting system consisting of a flapping foil and an electro-magnetic generator using power flow analysis. In: Proceedings of the ASME 2011 International Design Engineering Technical Conferences \& Computers and Information in Engineering Conference (IDETC/CIE 2011), Washington, DC, 1-8 (2011)

15. Xing, J.T., Xiong, Y.P., Wiercigroch, M., et al.: A mathematical modelling for an integrated electric converter-nonlinear oscillatorwater interaction system to harness wave energy. In: Proceedings of 7th European Nonlinear Dynamics Conference (ENOC 2011), Rome, Italy, 1-6 (2011) 
16. Xing, J.T.: Developments of numerical methods for linear and nonlinear fluid-solid interaction dynamics with applications. Adv. Mech. 46, 201602 (2016). doi:10.6052/1000-0992-15-038

17. Zuo, L., Scully, B., Shestani, J., et al.: Design and characterization of an electromagnetic energy harvester for vehicle suspensions. Smart Mater. Struct. 19, 045003 (2010). doi:10.1088/0964-1726/ 19/4/045003

18. Sharp, R., Crolla, D.: Road vehicle suspension system design-a review. Vehicle Syst. Dyn. 16, 167-192 (1987)

19. Karnopp, D.: Permanent magnet linear motors used as variable mechanical dampers for vehicle suspensions. Veh. Syst. Dyn. 18, 187-200 (1989)

20. Karnopp, D.: Power requirement for vehicle suspension systems. Veh. Syst. Dyn. 21, 65-71 (1992)

21. Segel, L., Lu, X.P.: Vehicular resistance to motion as influenced by road roughness and highway alignment. Aust. Road Res. 12, 211-222 (1982)

22. Hsu, P.: Power recovery property of electrical active suspension systems. In: Proceedings of the 31st Intersociety Energy Conversion Engineering Conference (IECEC 96), Washington, DC, 1899-1904 (1996)

23. Goldner, R., Zerigian, P., Hull, J.: A preliminary study of energy recovery in vehicles by using regenerative magnetic shock absorbers. SAE Technical Paper, 2001-01-2071 (2001). doi:10. 4271/2001-01-2071

24. Abouelnour, A., Hammad, N.: Electric utilization of vehicle damper dissipated energy. In: Al-Azhar Engineering Seventh International Conference (AEIC), Cairo Egypt, April 7-10 (2003)

25. Zheng, X., Yu, F.: Study on the potential benefits of an energyregenerative active suspension for vehicles. SAE Technical Paper, 2005-01-3564 (2005). doi:10.4271/2005-01-3564

26. Kawamoto, Y., Suda, Y., Inoue, H., et al.: Modelling of electromagnetic damper for automobile suspension. J. Syst. Des. Dyn. 1, 524-535 (2007)

27. Zhang, Y., Huang, K., Yu, F., et al.: Experimental verification of energy-regenerative feasibility for an automotive electrical suspension system. In: Proceedings of the IEEE International Conference on Vehicular Electronics and Safety, Beijing, December 13-15 (2007)

28. Priya, S.: Advances in energy harvesting using low profile piezoelectric transducers. J. Electroceram. 19, 165-182 (2007)

29. Zhang, Y., Yu, F., Huang, K.: A state of art review on regenerative vehicle active suspension. In: Proceedings of the 3rd International Conference on Mechanical Engineering and Mechanics (ICMEM), Beijing, October 21-23 (2009)
30. Montazeri-Gh, M., Soleymani, M.: Investigation of the energy regeneration of active suspension system in hybrid electric vehicles. IEEE Trans. Ind. Electron. 57, 918-925 (2010)

31. Ando, B., Baglio, S., Trigona, C., et al.: Nonlinear mechanism in MEMS devices for energy harvesting applications. J. Micromech. Microeng. 20, 125020 (2010). doi:10.1088/0960-1317/20/12/ 125020

32. Li, Z., Brindak, Z., Zuo, L.: Modeling of an electromagnetic vibration energy harvester with motion magnification. In: ASME 2011 International Mechanical Engineering Congress and Exposition, Denver, Colorado, 285-293 (2011)

33. Li, Z., Zuo, L., Kuang, J., et al.: Energy-harvesting shock absorber with a mechanical motion rectifier. Smart Mater. Struct. 22, 025008 (2013)

34. Zuo, L., Tang, X.: Large-scale vibration energy harvesting. J. Intell. Mater. Syst. Struct. 24, 1405-1430 (2013)

35. Li, Z., Zuo, L., Luhrs, G., et al.: Electromagnetic energy-harvesting shock absorbers: design, modeling, and road tests. IEEE Trans. Vehicul. Technol. 62, 1065 (2013)

36. Zuo, L., Zhang, P.: Energy harvesting, ride comfort and road handling of regenerative vehicle suspensions. J. Vib. Acoust. 133, 1-8 (2013)

37. Perpetuum: PMG17 vibration energy harvester-IEEE Xplore Digital Library. doi:10.1109/MCS.2007.911267

38. Xing, J.T.: Energy Flow Theory of Nonlinear Dynamical Systems with Applications. Springer, Heidelberg (2015)

39. Kittel, C.: Berkeley Physics Course. McGraw-Hill, New York (1967)

40. Xing, J.T., Xiong, Y.P., Price, W.G.: A generalized mathematical model and analysis for integrated multi-channel vibration structure-control interaction systems. J. Sound Vib. 322, 584-616 (2009)

41. Shim, T., Ghike, C.: Understanding the limitations of different vehicle models for roll dynamics studies. Veh. Syst. Dyn. 45, 191-216 (2007)

42. Bathe, K.J.: Finite Element Procedures. Prentice Hall, New Jersey (1996) 